\title{
Sampling-based Lower Bounds for Counting Queries
}

\author{
Vibhav Gogate \\ Computer Science \& Engineering \\ University of Washington \\ Seattle, WA 98195, USA \\ Email: vgogate@cs.washington.edu \\ Rina Dechter \\ Donald Bren School of Information and Computer Sciences \\ University of California, Irvine \\ Irvine, CA 92697, USA \\ Email: dechter@ics.uci.edu
}

\begin{abstract}
It is well known that computing relative approximations of weighted counting queries such as the probability of evidence in a Bayesian network, the partition function of a Markov network, and the number of solutions of a constraint satisfaction problem is NP-hard. In this paper, we settle therefore on an easier problem of computing highconfidence lower bounds and propose an algorithm based on importance sampling and Markov inequality for it. However, a straight-forward application of Markov inequality often yields poor lower bounds because it uses only one sample. We therefore propose several new schemes that extend it to multiple samples. Empirically, we show that our new schemes are quite powerful, often yielding substantially higher (better) lower bounds than state-of-the-art schemes.
\end{abstract}

\section{Introduction}

Many inference problems in graphical models such as finding the probability of evidence in a Bayesian network, the partition function of a Markov network, and the number of solutions of a constraint satisfaction problem are special cases of the following weighted counting problem: given a discrete function $F$, find the sum of $F$ over its domain. Therefore, efficient algorithms for computing the weighted counts are of paramount importance for a wide variety of applications that use graphical models, such as genetic linkage analysis (Fishelson and Geiger, 2003; Allen and Darwiche, 2008), car travel activity modeling (Liao et al., 2007; Gogate and Dechter, 2005), functional verification (Bergeron, 2000; Dechter et al., 2002a), target tracking (Pavlovic et al., 1999), machine vision (Fieguth et al., 1998; Li and Perona, 2005), medical diagnosis (Middleton et al., 1991; Pradhan et al., 1994) and music parsing (Raphael, 2002).

The weighted counting problem is in \#P and as a result there is no hope of designing efficient, general-purpose algorithms for it. Moreover, even approximations with relative error guarantees are NP-hard (Dagum and Luby, 1993). Therefore, previous work has focused either on approximations that have relative error guarantees for a restricted subclass of problems or on approximations with weaker guarantees such as bounding and convergence, whose good performance is demonstrated empirically. 
In this paper, we focus on general-purpose, lower bounding approximations of weighted counts and propose new randomized algorithms for it. An approximation algorithm is deterministic if it is always guaranteed to output a lower or an upper bound. On the other hand, an approximation algorithm is randomized if the approximation fails with a known probability $\delta \geq 0$. Lower bounds are useful because almost all applications of graphical models use probability thresholding. For instance, in medical diagnosis (Middleton et al., 1991; Pradhan et al., 1994), a disease diagnosis is made if the disease probability is greater than some threshold $t$. If the lower bound is greater than $t$, we are guaranteed that the actual probability is greater than $t$ too. Genetic linkage analysis (Ott, 1999; Fishelson and Geiger, 2003) is another example of an application where thresholds are used. Here, we are interested in knowing whether the likelihood of observing the test data given a particular value of linkage is greater than a threshold. If it is, then genetic linkage is said to have occurred.

Existing randomized, bounding algorithms (Cheng, 2001; Dagum and Luby, 1997) use known inequalities such as the Chebyshev and the Hoeffding inequalities (Hoeffding, 1963) to compute a relative approximation. These inequalities bound the deviation of the sample mean of $N$ independent random variables from the actual mean. The idea which is in some sense similar to importance sampling (Rubinstein, 1981; Geweke, 1989) is to express the weighted counting problem as the problem of computing the mean (or the expected value) of independent random variables and then use the mean over the sampled random variables to bound the deviation from the true mean. A serious limitation of these algorithms is that the number of samples required to guarantee high confidence bounds is inversely proportional to the weighted counts. Therefore, if the weighted counts are arbitrarily small (e.g., $\leq 10^{-20}$ ), a large number of samples (approximately $10^{19}$ ) are required to provide high confidence on the result.

We propose to alleviate this difficulty by using the Markov inequality which uses just one sample for lower bounding the weighted counts. The caveats are that we do not have relative error guarantees and the lower bound is quite weak because only one sample is used. To address this one-sample limitation, we propose to extend the Markov inequality to multiple samples. Recently, (Gomes et al., 2007) proposed to achieve this by using the minimum statistic. A major drawback of this approach is that as more samples are drawn, the minimum value will likely decrease and as a result the lower bound will decrease as well. To address this problem, we propose several new schemes that use the average, maximum and order statistics to improve the Markov inequality. Our new schemes guarantee that as more samples are drawn, the lower bound will likely increase.

We provide a thorough empirical evaluation demonstrating the potential of our new schemes. For the task of computing the probability of evidence, we compared against state-of-the-art deterministic approximations such as Variable elimination and Conditioning (VEC) (Dechter, 1999) and the active-tuples based (ATB) scheme (Bidyuk et al., 2010). For the task of counting the number of models of a satisfiability formula, we compared against Relsat (Bayardo and Pehoushek, 2000), which is a deterministic approximation and SampleCount (Gomes et al., 2007), which is a randomized algorithm. Our results clearly show that our new randomized approximations based on the Markov inequality are far more scalable than deterministic approximations such as VEC, Relsat and ATB, and in most cases yield far higher accuracy. Our schemes also yield higher lower bounds than SampleCount. ${ }^{1}$

The rest of this paper is organized as follows. In Section 2, we describe preliminaries

\footnotetext{
${ }^{1}$ The research presented in this paper is based in part on (Gogate et al., 2007).
} 
and previous work. In Section 3, we present our basic lower bounding scheme and several enhancements. Experimental results are presented in Section 4 and we conclude in Section 5 .

\section{Notation, Background and Previous work}

We denote variables by upper case letters (e.g., $X, Y, \ldots$ ) and values of variables by lower case letters (e.g., $x, y, \ldots$ ). Sets of variables are denoted by bold upper case letters (e.g., $\left.\mathbf{X}=\left\{X_{1}, \ldots, X_{n}\right\}\right)$. We denote the set of possible values (also called the domain) of $X_{i}$ by $D\left(X_{i}\right) . X_{i}=x_{i}$ (or simply $x_{i}$ when the variable is clear) denotes an assignment of a value $x_{i} \in D\left(X_{i}\right)$ to $X_{i}$ while $\mathbf{X}=\mathbf{x}$ (or simply $\mathbf{x}$ ) denotes an assignment of values to all variables in $\mathbf{X}$, namely $\mathbf{x}=\left(X_{1}=x_{1}, X_{2}=x_{2}, \ldots X_{n}=x_{n}\right) . D(\mathbf{X})$ denotes the Cartesian product of the domains of all variables in $\mathbf{X}$, namely $D(\mathbf{X})=D\left(X_{1}\right) \times \ldots \times D\left(X_{n}\right)$. The projection of $\mathbf{x}$ on a set $\mathbf{S} \subseteq \mathbf{X}$ is denoted by $\mathbf{x}_{\mathbf{S}}$. Given an assignment $\mathbf{y}$ and $\mathbf{z}$ to the partition $\mathbf{Y}$ and $\mathbf{Z}$ of $\mathbf{X}, \mathbf{x}=(\mathbf{y}, \mathbf{z})$ denotes their composition.

$\sum_{\mathbf{x} \in D(\mathbf{X})}$ denotes the sum over all possible configurations of variables in $\mathbf{X}$, namely, $\sum_{\mathbf{x} \in D(\mathbf{X})}=\sum_{x_{1} \in D\left(X_{1}\right)} \sum_{x_{2} \in D\left(X_{2}\right)} \cdots \times \sum_{x_{n} \in D\left(X_{n}\right)}$. For brevity, we will abuse notation and write $\sum_{x_{i} \in D\left(X_{i}\right)}$ as $\sum_{x_{i} \in X_{i}}$ and $\sum_{\mathbf{x} \in D(\mathbf{X})}$ as $\sum_{\mathbf{x} \in \mathbf{X}}$. The expected value $\operatorname{Ex}_{Q}[X]$ of a random variable $X$ with respect to a distribution $Q$ is defined as: $\operatorname{Ex}_{Q}[X]=\sum_{x \in X} x Q(x)$. The variance $\operatorname{Var}_{Q}[X]$ of $X$ is defined as: $\operatorname{Var}_{Q}[X]=\sum_{x \in X}\left(x-\operatorname{Ex}_{Q}[X]\right)^{2}$. To simplify, we will write $\operatorname{Ex}_{Q}[X]$ as $\operatorname{Ex}[X]$ and $\operatorname{Var}_{Q}[X]$ as $\operatorname{Var}[X]$, when the identity of $Q$ is clear.

We denote (discrete) functions by upper case letters (e.g. $F, H, C, I$ etc.), and the scope (set of arguments) of a function $F$ by $V(F)$. Given an assignment $\mathbf{y}$ to a superset $\mathbf{Y}$ of $V(F)$, we will abuse notation and write $F\left(\mathbf{y}_{V(F)}\right)$ as $F(\mathbf{y})$.

Definition 1. A discrete graphical model or a Markov network denoted by $\mathcal{G}$ is a 3tuple $\langle\mathbf{X}, \mathbf{D}, \mathbf{F}\rangle$ where $\mathbf{X}=\left\{X_{1}, \ldots, X_{n}\right\}$ is a finite set of variables, $\mathbf{D}=\left\{D\left(X_{1}\right), \ldots, D\left(X_{n}\right)\right\}$ is a finite set of domains where $D\left(X_{i}\right)$ is the domain of variable $X_{i}$ and $\mathbf{F}=\left\{F_{1}, \ldots, F_{m}\right\}$ is a finite set of discrete-valued non-negative functions (also called potentials). The graphical model represents a joint distribution $P_{\mathcal{G}}$ over $\mathbf{X}$ defined as:

$$
P_{\mathcal{G}}(\mathbf{x})=\frac{1}{Z} \prod_{i=1}^{m} F_{i}(\mathbf{x})
$$

where $Z$ is a normalization constant, often called the partition function. It is given by:

$$
Z=\sum_{\mathbf{x} \in \mathbf{X}} \prod_{i=1}^{m} F_{i}(\mathbf{x})
$$

The primary queries over Markov networks are computing the partition function and computing the marginal probability $P_{\mathcal{G}}\left(X_{i}=x_{i}\right)$. The weighted counting problem is to compute the weighted counts $Z$.

Each graphical model is associated with a primal graph which depicts the dependencies between its variables.

Definition 2. The primal graph of a graphical model $\mathcal{G}=\langle\mathbf{X}, \mathbf{D}, \mathbf{F}\rangle$ is an undirected graph $G(\mathbf{X}, \mathbf{E})$ which has variables of $\mathcal{G}$ as its vertices and an edge between two variables that appear in the scope of a function. 


\subsection{Bayesian and Constraint networks}

Definition 3. A Bayesian network is a graphical model $\mathcal{B}=\langle\mathbf{X}, \mathbf{D}, \mathbf{G}, \mathbf{P}\rangle$ where $G=$ $(\mathbf{X}, \mathbf{E})$ is a directed acyclic graph over the set of variables $\mathbf{X}$. Each function $P_{i} \in \mathbf{P}$ is a conditional probability table defined as $P_{i}\left(X_{i} \mid \mathbf{p} \mathbf{a}_{i}\right)$, where $\mathbf{p} \mathbf{a}_{i}=V\left(P_{i}\right) \backslash\left\{X_{i}\right\}$ is the set of parents of $X_{i}$ in $G$.

The primal graph of a Bayesian network is also called the moral graph. When the entries of the CPTs are 0 and 1 only, they are called deterministic or functional CPTs. An evidence $\mathbf{E}=\mathbf{e}$ is an instantiated subset of variables. A Bayesian network represents the following joint probability distribution:

$$
P_{\mathcal{B}}(\mathbf{x})=\prod_{i=1}^{n} P_{i}\left(\mathbf{x}_{\left\{X_{i}\right\}} \mid \mathbf{x}_{\mathbf{p a}_{i}}\right)
$$

By definition, given a Bayesian network $\mathcal{B}$ the probability of evidence $P_{\mathcal{B}}(\mathbf{e})$ is given by:

$$
P_{\mathcal{B}}(\mathbf{e})=\sum_{\mathbf{y} \in \mathbf{X} \backslash \mathbf{E}} \prod_{i=1}^{n} P_{i}\left((\mathbf{y}, \mathbf{e})_{\left\{X_{i}\right\}} \mid(\mathbf{y}, \mathbf{e})_{\mathbf{p a}_{i}}\right)
$$

It is easy to see from Equations 2 and 4 that $P_{\mathcal{B}}(\mathbf{e})$ is equivalent to the weighted counts $Z$ over an evidence instantiated Bayesian network. Another important query over a Bayesian network is computing the conditional marginal probability $P_{\mathcal{B}}\left(x_{i} \mid \mathbf{e}\right)$ for a query variable $X_{i} \in \mathbf{X} \backslash \mathbf{E}$.

Definition 4. A constraint network is a graphical model $\mathcal{R}=\langle\mathbf{X}, \mathbf{D}, \mathbf{C}\rangle$ where $\mathbf{C}=$ $\left\{C_{1}, \ldots, C_{m}\right\}$ is a set of constraints. Each constraint $C_{i}$ is a $0 / 1$ function defined over its scope. Given an assignment $\mathbf{x}$, a constraint is said to be satisfied if $C_{i}(\mathbf{x})=1$. A constraint can also be expressed by a pair $\left\langle R_{i}, \mathbf{S}_{i}\right\rangle$ where $R_{i}$ is a relation defined over the scope of $C_{i}$ that contains all tuples for which $C_{i}\left(\mathbf{s}_{i}\right)=1$. The primal graph of a constraint network is called the constraint graph.

A solution of a constraint network is an assignment $\mathbf{x}$ to all variables that satisfies all the constraints. The primary query over a constraint network is to determine whether it has a solution and if it does to find one. Another important query is that of counting the number of solutions $K$ of the constraint network, defined by:

$$
K=\sum_{\mathbf{x} \in \mathbf{X}} \prod_{i=1}^{m} C_{i}(\mathbf{x})
$$

$K$ is clearly identical to the weighted counts over a constraint network.

\subsection{Previous work}

Earlier work by Dagum and Luby (Dagum and Luby, 1997) and by Cheng (Cheng, 2001) on randomized bounding algorithms for weighted counting has focused on providing relativeerror guarantees. Their algorithms are based on importance sampling (Marshall, 1956). The main idea in importance sampling is to express the weighted counts as an expectation using an easy-to-sample distribution $Q$, which is called the proposal (or trial or importance) distribution. Then, the algorithm generates samples from $Q$ and estimates the expectation 
(which equals the weighted counts) by a weighted average over the samples, where the weight of a sample $\mathbf{x}$ is $\prod_{i=1}^{m} F_{i}(\mathbf{x}) / Q(\mathbf{x})$. The weighted average is often called the sample mean.

Formally, given a proposal distribution $Q$ such that $\prod_{i=1}^{m} F_{i}(\mathbf{x}) \geq 0 \Rightarrow Q(\mathbf{x}) \geq 0$, we can rewrite Equation 2 as follows:

$$
Z=\sum_{\mathbf{x} \in \mathbf{X}} \frac{\prod_{i=1}^{m} F_{i}(\mathbf{x})}{Q(\mathbf{x})} Q(\mathbf{x})=\operatorname{Ex}_{Q}\left[\frac{\prod_{i=1}^{m} F_{i}(\mathbf{x})}{Q(\mathbf{x})}\right]
$$

Given independent and identically distributed (i.i.d.) samples $\left(\mathbf{x}^{1}, \ldots, \mathbf{x}^{N}\right)$ generated from $Q$, we can estimate $Z$ by:

$$
\widehat{Z}_{N}=\frac{1}{N} \sum_{k=1}^{N} \frac{\prod_{i=1}^{m} F_{i}\left(\mathbf{x}^{k}\right)}{Q\left(\mathbf{x}^{k}\right)}=\frac{1}{N} \sum_{k=1}^{N} w\left(\mathbf{x}^{k}\right)
$$

where

$$
w(\mathbf{x})=\frac{\prod_{i=1}^{m} F_{i}(\mathbf{x})}{Q(\mathbf{x})}
$$

is the weight of sample $\mathbf{x}$. It is easy to show that $\widehat{Z}_{N}$ is unbiased, namely $\operatorname{Ex}_{Q}\left[\widehat{Z}_{N}\right]=Z$.

Dagum and Luby (Dagum and Luby, 1997) provide a bound on the number of samples $N$ required to guarantee that for any $\epsilon, \delta \geq 0$, the estimate $\widehat{Z}_{N}$ approximates $Z$ with relative error $\epsilon$ with probability at least $1-\delta$. Formally,

$$
\operatorname{Pr}\left[Z(1-\epsilon) \leq \widehat{Z}_{N} \leq Z(1+\epsilon)\right] \geq 1-\delta
$$

when $N$ satisfies:

$$
N \geq \frac{4}{Z \epsilon^{2}} \ln \frac{2}{\delta}
$$

This bound was later improved by Cheng (Cheng, 2001) yielding:

$$
N \geq \frac{1}{Z} \frac{1}{(1+\epsilon) \ln (1+\epsilon)-\epsilon} \ln \frac{2}{\delta}
$$

In both of these bounds (see Equations 9 and 10 ) $N$ is inversely proportional to $Z$ and therefore when $Z$ is small, a large number of samples are required to achieve an acceptable confidence level $(1-\delta) \geq 0.99$.

A bound on $N$ is required because (Dagum and Luby, 1997; Cheng, 2001) insist on a relative error $\epsilon$. If we relax this requirement and if we use the Markov inequality, even a single sample would yield a high confidence lower bound on $Z$. Furthermore, the lower bound can be improved with more samples, as we demonstrate in the next section.

\section{Markov Inequality based Lower Bounds}

Proposition 1 (Markov Inequality). For any random variable $X$ and a real number $r \geq 1$, $\operatorname{Pr}(X \geq r \mathbb{E}[X]) \leq \frac{1}{r}$.

The Markov inequality states that the probability that a random variable is $r$ times its expected value is less than or equal to $1 / r$. 
We can apply the Markov inequality for lower bounding the weighted counts in a straight-forward manner. We can consider the weight of each sample generated by importance sampling as a random variable. Because the expected value of the weight equals the weighted counts $Z$, by Markov inequality, given a real number $r \geq 1$, the probability that the weight of a sample is greater than $r$ times $Z$ is less than $1 / r$. Alternately, the weight of the sample divided by $r$ is a lower bound on $Z$ with probability greater than $1-1 / r$. Formally, given a sample $\mathbf{x}$ drawn independently from a proposal distribution $Q$, we have:

$$
\operatorname{Pr}(w(\mathbf{x}) \geq r \times Z) \leq \frac{1}{r}
$$

Rearranging Equation 11, we get:

$$
\operatorname{Pr}\left(\frac{w(\mathbf{x})}{r} \leq Z\right) \geq 1-\frac{1}{r}
$$

Equation 12 can be used to probabilistically lower bound $Z$ as shown in the following example.

Example 1. Let $r=100$ and let $\mathrm{x}$ be a sample generated using importance sampling. Then from Equation 12, $\frac{w(\mathbf{x})}{100}$ is a lower bound on $Z$ with probability greater than $1-(1 / 100)=$ 0.99 .

The lower bound based on the Markov inequality uses just one sample and is therefore likely to be very weak. In the following four subsections, we show how the lower bounds can be improved by utilizing multiple samples.

\subsection{The Minimum scheme}

The Minimum scheme (Gomes et al., 2007) uses the minimum over the sample weights to compute a lower bound on $Z$. Although, originally introduced in the context of lower bounding the number of solutions of a Boolean satisfiability (SAT) problem, we can easily modify it to compute a lower bound on the weighted counts as we show next.

Theorem 1 (minimum scheme). Given $N$ samples $\left(\boldsymbol{x}^{1}, \ldots, \boldsymbol{x}^{N}\right)$ drawn independently from a proposal distribution $Q$ such that $\mathbb{E}\left[w\left(\boldsymbol{x}^{i}\right)\right]=Z$ for $i=1, \ldots, N$ and a constant $0 \leq \alpha \leq 1$

$$
\boldsymbol{P r}\left[\min _{i=1}^{N}\left[\frac{w\left(\mathbf{x}^{i}\right)}{\beta}\right] \leq Z\right] \geq \alpha, \text { where } \beta=\left(\frac{1}{1-\alpha}\right)^{\frac{1}{N}}
$$

Proof. Consider an arbitrary sample $\mathbf{x}^{i}$. From the Markov inequality, we get:

$$
\operatorname{Pr}\left[\frac{w\left(\mathbf{x}^{i}\right)}{\beta} \geq Z\right] \leq \frac{1}{\beta}
$$

Since, the generated $N$ samples are independent, the probability that the minimum over them is also an upper bound is given by:

$$
\operatorname{Pr}\left[\min _{i=1}^{N}\left[\frac{w\left(\mathbf{x}^{i}\right)}{\beta}\right] \geq Z\right] \leq \frac{1}{\beta^{N}}
$$




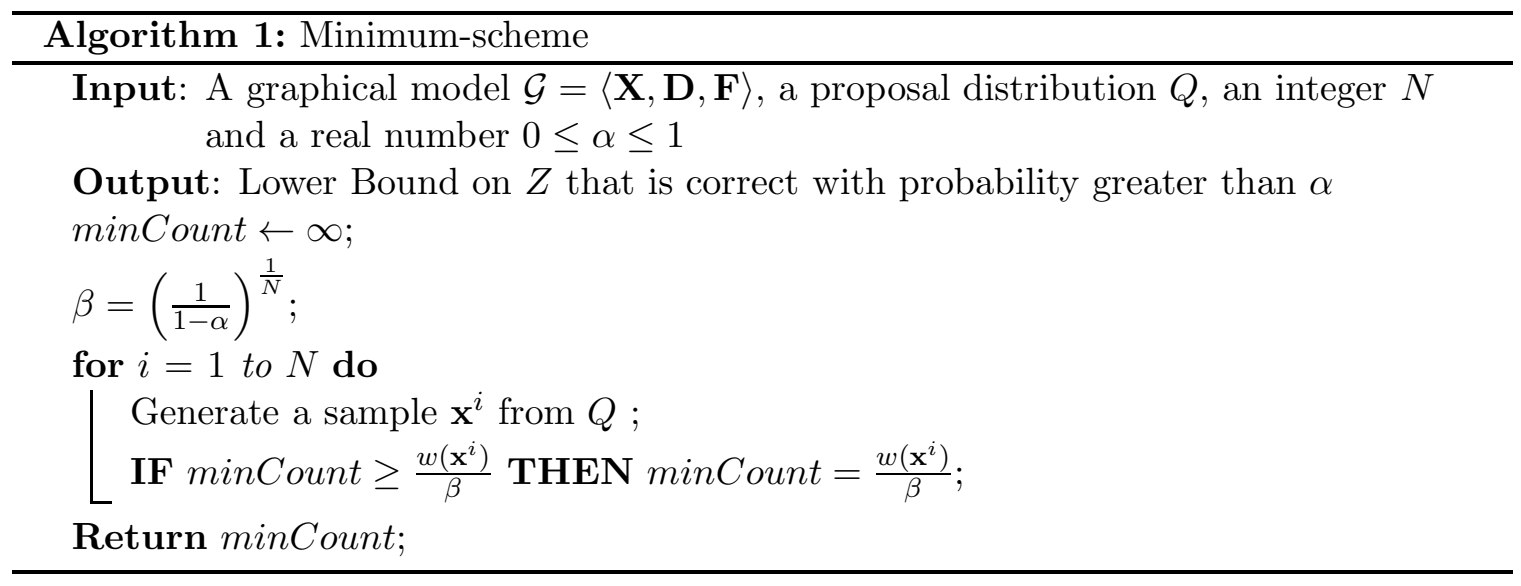

Rearranging Equation 14, we get:

$$
\operatorname{Pr}\left[\min _{i=1}^{N}\left[\frac{w\left(\mathbf{x}^{i}\right)}{\beta}\right] \leq Z\right] \geq 1-\frac{1}{\beta^{N}}
$$

Substituting $\beta=\left(\frac{1}{1-\alpha}\right)^{\frac{1}{N}}$ in $1-\frac{1}{\beta^{N}}$, we get:

$$
\begin{aligned}
1-\frac{1}{\beta^{N}} & =1-\frac{1}{\left(\left(\frac{1}{1-\alpha}\right)^{\frac{1}{N}}\right)^{N}} \\
& =1-\frac{1}{\frac{1}{1-\alpha}} \\
& =1-(1-\alpha) \\
& =\alpha
\end{aligned}
$$

Therefore, from Equations 15 and 16, we have

$$
\operatorname{Pr}\left[\min _{i=1}^{k}\left[\frac{w\left(\mathbf{x}^{i}\right)}{\beta}\right] \leq Z\right] \geq \alpha
$$

Algorithm 1 describes the minimum scheme based on Theorem 1. The algorithm first calculates $\beta$ based on the value of $\alpha$ and $N$. It then returns the minimum of $\frac{w\left(\mathbf{x}^{i}\right)}{\beta}$ (minCount in Algorithm 1) over the $N$ samples.

A nice property of the minimum scheme is that with more samples the divisor, $\beta=$ $\frac{1}{(1-\alpha)^{\frac{1}{N}}}$ decreases, thereby (possibly) increasing the lower bound. The problem is that because it computes a minimum over the sample weights, we expect the lower bound to decrease when the number of samples increases, unless the variance of the weights is very small. Next, we present our first contribution, the average scheme, which avoids this problem. 


\subsection{The Average Scheme}

An obvious scheme is to use the unbiased importance sampling estimator $\widehat{Z}_{N}$ given in

Equation 7. Because $\mathbb{E}_{Q}\left[\widehat{Z}_{N}\right]=Z$, from the Markov inequality $\frac{\widehat{Z}_{N}}{\beta}$ where $\beta=\frac{1}{1-\alpha}$ is a lower bound of $Z$ with probability greater than $\alpha$. Formally,

$$
\operatorname{Pr}\left[\frac{\widehat{Z}_{N}}{\beta} \leq Z\right] \geq \alpha, \text { where } \beta=\frac{1}{1-\alpha}
$$

As more samples are drawn the average is likely to be get larger than the minimum value, increasing the lower bound. However, unlike the minimum scheme in which the divisor $\beta$ decreases with an increase in the sample size thereby increasing the lower bound, the divisor $\beta$ in the average scheme remains constant. As a consequence, for example, if all the generated samples have the same weight (or almost the same weight), the lower bound due to the minimum scheme would be greater than the lower bound output by the average scheme. In practice the variance is typically never close to zero and therefore the average scheme is likely to be superior.

\subsection{The Maximum scheme}

We can also use the maximum instead of the average over the $N$ i.i.d samples as shown in the following Lemma.

Lemma 1 (maximum scheme). Given $N$ samples $\left(\mathbf{x}^{1}, \ldots, \mathbf{x}^{N}\right)$ drawn independently from a proposal distribution $Q$ such that $\mathbb{E}\left[w\left(\mathbf{x}^{i}\right)\right]=Z$ for $i=1, \ldots, N$ and a constant $0 \leq \alpha \leq 1$,

$$
\operatorname{Pr}\left[\frac{\max _{i=1}^{N} w\left(\mathbf{x}^{i}\right)}{\beta} \leq Z\right] \geq \alpha, \text { where } \beta=\frac{1}{1-\alpha^{\frac{1}{N}}}
$$

Proof. From Markov inequality, we have:

$$
\operatorname{Pr}\left[\frac{w\left(\mathbf{x}^{i}\right)}{\beta} \leq Z\right] \geq 1-\frac{1}{\beta}
$$

Given a set of $N$ independent events such that each event occurs with probability $\geq$ $(1-1 / \beta)$, the probability that all events occur is $\geq(1-1 / \beta)^{N}$. In other words, given $N$ independent samples such that the weight of each sample divided by $\beta$ is a lower bound on $Z$ with probability $\geq(1-1 / \beta)$, the probability that the weights of all samples divided by $\beta$ are a lower bound on $Z$ is $\geq(1-1 / \beta)^{N}$. Consequently,

$$
\operatorname{Pr}\left[\frac{\max _{i=1}^{N} w\left(\mathbf{x}^{i}\right)}{\beta} \leq Z\right] \geq\left(1-\frac{1}{\beta}\right)^{N}
$$

Substituting the value of $\beta$ in $\left(1-\frac{1}{\beta}\right)^{N}$, we have:

$$
\begin{aligned}
\left(1-\frac{1}{\beta}\right)^{N} & =\left(1-\frac{1}{\frac{1}{1-\alpha^{\frac{1}{N}}}}\right)^{N} \\
& =\left(1-\left(1-\alpha^{\frac{1}{N}}\right)\right)^{N} \\
& =\alpha
\end{aligned}
$$


From Equations 20 and 21, we get:

$$
\operatorname{Pr}\left[\frac{\max _{i=1}^{N} w\left(\mathbf{x}^{i}\right)}{\beta} \leq Z\right] \geq \alpha
$$

The problem with the maximum scheme is that increasing the number of samples increases $\beta$ and consequently the lower bound decreases. However, when only a few samples are available and the variance of the weights $w\left(\mathbf{x}^{i}\right)$ is large, the maximum value is likely to be larger than the sample average and obviously the minimum.

\subsection{Using the Martingale Inequalities}

Another approach to utilize the maximum over the $N$ samples is to use the martingale inequalities.

Definition 5 (Martingale). A sequence of random variables $X_{1}, \ldots, X_{N}$ is a martingale with respect to another sequence $Y_{1}, \ldots, Y_{N}$ defined on a common probability space $\Omega$ iff $\mathbb{E}\left[X_{i} \mid Y_{1}, \ldots, Y_{i-1}\right]=X_{i-1}$ for all $i$.

It is easy to see that given i.i.d. samples $\left(\mathbf{x}^{1}, \ldots, \mathbf{x}^{N}\right)$ generated from $Q$, the sequence $\Lambda_{1}, \ldots, \Lambda_{N}$, where $\Lambda_{p}=\prod_{i=1}^{p} \frac{w\left(\mathbf{x}^{i}\right)}{Z}$ forms a martingale as shown below:

$$
\begin{aligned}
\mathbb{E}\left[\Lambda_{p} \mid \mathbf{x}^{1}, \ldots, \mathbf{x}^{p-1}\right] & =\mathbb{E}\left[\Lambda_{p-1} * \frac{w\left(\mathbf{x}^{p}\right)}{Z} \mid \mathbf{x}^{1}, \ldots, \mathbf{x}^{p-1}\right] \\
& =\Lambda_{p-1} * \mathbb{E}\left[\frac{w\left(\mathbf{x}^{p}\right)}{Z} \mid \mathbf{x}^{1}, \ldots, \mathbf{x}^{p-1}\right]
\end{aligned}
$$

Because $\mathbb{E}\left[\frac{w\left(\mathbf{x}^{p}\right)}{Z} \mid \mathbf{x}^{1}, \ldots, \mathbf{x}^{p-1}\right]=1$, we have $\mathbb{E}\left[\Lambda_{p} \mid \mathbf{x}^{1}, \ldots, \mathbf{x}^{p-1}\right]=\Lambda_{p-1}$ as required. The expected value $\mathbb{E}\left[\Lambda_{1}\right]=1$ and for such martingales which have a mean of 1 , Breiman (Breiman, 1968) provides the following extension of the Markov inequality:

$$
\operatorname{Pr}\left(\max _{i=1}^{N} \Lambda_{i} \geq \beta\right) \leq \frac{1}{\beta}
$$

and therefore,

$$
\operatorname{Pr}\left(\left[\max _{i=1}^{N} \prod_{j=1}^{i} \frac{w\left(\mathbf{x}^{j}\right)}{Z}\right] \geq \beta\right) \leq \frac{1}{\beta}
$$

From Inequality 24, we can prove that:

Theorem 2 (Random permutation scheme). Given $N$ samples $\left(\mathbf{x}^{1}, \ldots, \mathbf{x}^{N}\right)$ drawn independently from a proposal distribution $Q$ such that $\mathbb{E}\left[w\left(\mathbf{x}^{i}\right)\right]=Z$ for $i=1, \ldots, N$ and a constant $0 \leq \alpha \leq 1$,

$$
\operatorname{Pr}\left[\max _{i=1}^{N}\left(\frac{1}{\beta} \prod_{j=1}^{i} w\left(\mathbf{x}^{j}\right)\right)^{1 / i} \leq Z\right] \geq \alpha, \text { where } \beta=\frac{1}{1-\alpha}
$$


Proof. From Inequality 24, we have:

$$
\operatorname{Pr}\left(\left[\max _{i=1}^{N} \prod_{j=1}^{i} \frac{w\left(\mathbf{x}^{j}\right)}{Z}\right] \geq \beta\right) \leq \frac{1}{\beta}
$$

Rearranging Inequality 25, we have:

$$
\operatorname{Pr}\left[\max _{i=1}^{N}\left(\frac{1}{\beta} \prod_{j=1}^{i} w\left(\mathbf{x}^{j}\right)\right)^{1 / i} \leq Z\right] \geq 1-\frac{1}{\beta}=\alpha
$$

Therefore, given $N$ samples, the following quantity

$$
\max _{i=1}^{N}\left(\frac{1}{\beta} \prod_{j=1}^{i} w\left(\mathbf{x}^{j}\right)\right)^{1 / i} \quad \text { where } \beta=\frac{1}{1-\alpha}
$$

is a lower bound on $Z$ with a confidence greater than $\alpha$. In general one could use any randomly selected permutation of the samples $\left(\mathbf{x}^{1}, \ldots, \mathbf{x}^{N}\right)$ and apply inequality 24 . We therefore call this scheme as the random permutation scheme.

Another related extension of Markov inequality for martingales deals with the order statistics of the samples. Let $\frac{w\left(\mathbf{x}^{(1)}\right)}{Z} \leq \frac{w\left(\mathbf{x}^{(2)}\right)}{Z} \leq \ldots \leq \frac{w\left(\mathbf{x}^{(N)}\right)}{Z}$ be the order statistics of the sample. Using martingale theory, Kaplan (Kaplan, 1987) proved that the random variable

$$
\Theta^{*}=\max _{i=1}^{N} \prod_{j=1}^{i} \frac{w\left(\mathbf{x}^{(N-j+1)}\right)}{Z \times\left(\begin{array}{c}
N \\
i
\end{array}\right)}
$$

satisfies the inequality $\operatorname{Pr}\left(\Theta^{*} \geq k\right) \leq 1 / k$. Therefore,

$$
\operatorname{Pr}\left(\left[\max _{i=1}^{N} \prod_{j=1}^{i} \frac{w\left(\mathbf{x}^{(N-j+1)}\right)}{Z \times\left(\begin{array}{c}
N \\
i
\end{array}\right)}\right] \geq \beta\right) \leq \frac{1}{\beta}
$$

From Inequality 27, we can prove that:

Theorem 3 (Order Statistics scheme). Given an order statistics of the weights $\frac{w\left(\mathbf{x}^{(1)}\right)}{Z} \leq$ $\frac{w\left(\mathbf{x}^{(2)}\right)}{Z} \leq \ldots \leq \frac{w\left(\mathbf{x}^{(N)}\right)}{Z}$ of $N$ samples $\left(\mathbf{x}^{1}, \ldots, \mathbf{x}^{N}\right)$ drawn independently from a proposal distribution $Q$, such that $\mathbb{E}\left[w\left(\mathbf{x}^{i}\right)\right]=Z$ for $i=1, \ldots, N$ and a constant $0 \leq \alpha \leq 1$,

$$
\operatorname{Pr}\left[\max _{i=1}^{N}\left(\frac{1}{\beta} \prod_{j=1}^{i} \frac{w\left(\mathbf{x}^{(N-j+1)}\right)}{\left(\begin{array}{c}
N \\
i
\end{array}\right)}\right)^{1 / i} \leq Z\right] \geq \alpha \text {, where } \beta=\frac{1}{1-\alpha}
$$

Proof. From Inequality 27, we have:

$$
\operatorname{Pr}\left(\left[\max _{i=1}^{N} \prod_{j=1}^{i} \frac{w\left(\mathbf{x}^{(N-j+1)}\right)}{Z \times\left(\begin{array}{c}
N \\
i
\end{array}\right)}\right] \geq \beta\right) \leq \frac{1}{\beta}
$$


Rearranging Inequality 28, we have:

$$
\operatorname{Pr}\left[\max _{i=1}^{N}\left(\frac{1}{\beta} \prod_{j=1}^{i} \frac{w\left(\mathbf{x}^{(N-j+1)}\right)}{\left(\begin{array}{c}
N \\
i
\end{array}\right)}\right)^{1 / i} \leq Z\right] \geq 1-\frac{1}{\beta}=\alpha
$$

Thus, given $N$ samples, the following quantity

$$
\max _{i=1}^{N}\left(\frac{1}{\beta} \prod_{j=1}^{i} \frac{w\left(\mathbf{x}^{(N-j+1)}\right)}{\left(\begin{array}{c}
N \\
i
\end{array}\right)}\right)^{1 / i}, \text { where } \beta=\frac{1}{1-\alpha}
$$

is a lower bound on $Z$ with probability greater than $\alpha$. Because the lower bound is based on the order statistics, we call this scheme as the order statistics scheme.

To summarize, we described five schemes that generalize the Markov inequality to multiple samples: (1) The minimum scheme (Gomes et al., 2007), (2) The average scheme, (3) The maximum scheme, (4) The martingale random permutation scheme and (5) The martingale order statistics scheme. All these schemes can be used with any sampling scheme that outputs unbiased sample weights to yield a probabilistic lower bound on the weighted counts.

\section{Empirical Evaluation}

In this section, we compare the performance of the probabilistic lower bounding schemes presented in this paper with other deterministic schemes from literature. We also evaluate the relative performance of the various lower bounding schemes presented in Section 3. We conducted experiments on three weighted counting tasks: (a) Satisfiability model counting, (b) computing probability of evidence in a Bayesian network and (c) computing the partition function of a Markov network. Our experimental data clearly demonstrates that our new lower bounding schemes are more accurate, robust and scalable than all other deterministic approximations, yielding far better (higher) lower bounds on large, hard instances.

\subsection{The Algorithms Evaluated}

We experimented with the following five schemes.

1. Variable Elimination and Conditioning (VEC). When a problem having a high treewidth is encountered, bucket elimination ${ }^{2}$ (Dechter, 1999) may be unsuitable, primarily because of its extensive memory demand. To alleviate this limitation, (Dechter, 1999; Rish and Dechter, 2000; Larrosa and Dechter, 2003) proposed the $w$-cutset conditioning scheme. The main idea is to condition or instantiate enough variables (the $w$-cutset) such that the remaining problem, after removing the instantiated variables, can be solved exactly using bucket elimination. Formally, given an integer bound $w$, we partition the variables into two subsets $\mathbf{K}$ and $\mathbf{R}$ such that the treewidth of the graphical model restricted to $\mathbf{R}$ is bounded

\footnotetext{
${ }^{2}$ Bucket elimination is an exact algorithm for computing the weighted counts. Its time and space complexity is exponential in the treewidth.
} 
by $w$ ( $\mathbf{K}$ is called the w-cutset). Then, we compute the weighted counts by summing over the exact solution output by bucket elimination for all possible instantiations $\mathbf{K}=\mathbf{k}$ of the $w$-cutset. We call this scheme variable elimination and conditioning (VEC). If VEC is terminated before completion, it outputs a partial sum yielding a lower bound on the weighted counts.

For VEC, our design choice is to select the $w$-cutset such that bucket elimination would require less than $1.5 \mathrm{~GB}$ of space. This is done to ensure that bucket elimination terminates in a reasonable amount of time and uses bounded space.

For models having determinism, as pre-processing, we use a SAT solver for removing all inconsistent values from the domains of all variables. We found that this pre-processing step yields significant performance gains in practice (for details, see the results of UAI 2008 (Darwiche et al., 2008) and UAI 2010 (Elidan and Globerson, 2010) competitions). We now briefly describe how we implemented this pre-processing. We first encode all the zero probabilities in the graphical model as a CNF formula $F$ (this can be done, for example, using the direct encoding of (Walsh, 2000)). Then, for each variable-value pair $(X, x)$, we construct a new $\mathrm{CNF}$ formula $F_{X, x}$ by adding a unit clause corresponding to $X=x$ to $F$ and check using a SAT solver (we used the minisat solver (Sorensson and Een, 2005) in our implementation) whether $F_{X, x}$ is consistent or not. If $F_{X, x}$ is inconsistent then the assignment $X=x$ is inconsistent and we delete $x$ from the domain of $X$.

The implementation of VEC is available publicly from our software web page (Dechter et al., 2009).

2. Active-Tuples based scheme We also experimented with the state of the art anytime bounding scheme (Bidyuk et al., 2010) that combines sampling-based w-cutset conditioning and bound propagation (Leisink and Kappen, 2003). As mentioned earlier, given a w-cutset $\mathbf{K} \subseteq \mathbf{X}$, we can compute the weighted counts exactly as follows:

$$
Z=\sum_{\mathbf{k} \in \mathbf{K}} Z(\mathbf{k})
$$

where $Z(\mathbf{k})=\sum_{\mathbf{r} \in \mathbf{R}} \prod_{i=1}^{m} F_{i}(\mathbf{r}, \mathbf{k})$. The lower bound on $Z$ is obtained by computing $Z(\mathbf{k})$ for $h$ high probability tuples of $\mathbf{K}$ (selected through sampling) and lower bounding the remaining probability mass using bound propagation (Leisink and Kappen, 2003). Formally, let $\left(\mathbf{k}^{1}, \ldots, \mathbf{k}^{m}\right)$ denote all the tuples $D(\mathbf{K})$. Given $h$ w-cutset tuples, $0 \leq h \leq m$, that we assume without loss of generality to be the first $h$ tuples according to some enumeration order, we can rewrite Equation 30 as:

$$
Z=\sum_{i=1}^{h} Z\left(\mathbf{k}^{i}\right)+Z_{B d P}^{L}\left(\mathbf{k}^{h+1: m}\right)
$$

where $Z_{B d P}^{L}\left(\mathbf{k}^{h+1: m}\right)$ is a lower bound on $\sum_{i=h+1}^{m} Z\left(\mathbf{k}^{i}\right)$, obtained using bound propagation. The lower bound obtained by Equation 31 can be improved by exploring a larger number of tuples $h$ thus relying on exact computation and less on the bounding scheme. In our experiments we run the bound propagation with w-cutset conditioning scheme until convergence or until a stipulated time bound has expired. Note that the bound propagation with cutset conditioning scheme provides deterministic lower and upper bounds on the weighted counts while the schemes presented in this paper only provide a probabilistic lower bound. 
3. Markov-LB with SampleSearch and IJGP-sampling. Recall that our Markov inequality based lower bounding schemes described in Section 3 can be combined with any importance sampling algorithm (henceforth, we will call them Markov-LB). In order to compete and compare fairly with existing algorithms, we apply Markov-LB on top of state-of-the-art importance sampling techniques such as IJGP-IS (Gogate and Dechter, 2005; Gogate, 2009) and IJGP-SampleSearch (Gogate and Dechter, 2011). Both of these are importance sampling schemes that sample from a proposal distribution generated from the output of a generalized belief propagation (Yedidia et al., 2004) algorithm. IJGPSampleSearch utilizes, in addition, a scheme to manage rejection when the probability distribution contains significant amount of determinism. We provide some more details and background next.

IJGP-IS constructs the proposal distribution using the output of a generalized belief propagation scheme called Iterative Join Graph Propagation (IJGP) (Dechter et al., 2002b; Mateescu et al., 2010). It was shown that belief propagation schemes whether applied over the original graph or on clusters of nodes yield a better approximation to the true posterior than other available choices (Mateescu et al., 2010; Murphy et al., 1999; Yedidia et al., 2004) and thus could lead to a better proposal distribution (see (Yuan and Druzdzel, 2006; Gogate and Dechter, 2005; Gogate, 2009) for more details).

IJGP (Dechter et al., 2002b; Mateescu et al., 2010) is a generalized belief propagation scheme which is parametrized by an $i$-bound, yielding a class of algorithms $\operatorname{IJGP}(i)$ whose complexity is exponential in $i$, that trade-off accuracy and complexity. As $i$ increases, accuracy generally increases. In our experiments, for every instance, we select the maximum $i$-bound that can be accommodated by $512 \mathrm{MB}$ of space as follows. The space required by a message (or a function) is the product of the domain sizes of the variables in its scope. Given an $i$-bound, we can create a join graph whose cluster size is bounded by $i$ as described in (Mateescu et al., 2010) and compute, in advance, the space required by IJGP by summing over the space required by the individual messages. ${ }^{3}$ We iterate from $i=1$ until the space bound (of $512 \mathrm{MB}$ ) is surpassed. This ensures that IJGP terminates in a reasonable amount of time and requires bounded space.

On networks having substantial amount of determinism, we use IJGP-based SampleSearch (IJGP-SS) (Gogate and Dechter, 2007, 2011). It is known that on such networks pure importance sampling generates many useless zero weight samples which are eventually rejected. SampleSearch overcomes this rejection problem by explicitly searching for a non-zero weight sample, yielding a more efficient sampling scheme in heavily deterministic databases. It was shown that SampleSearch is an importance sampling scheme which generates samples from a modification of the proposal distribution which is backtrack-free w.r.t. the constraints. Thus, in order to derive the weights of the samples generated by SampleSearch, all we need is to replace the proposal distribution with the backtrack-free distribution. The details are given in (Gogate and Dechter, 2011).

To reduce the variance of the weights, we combine both IJGP-IS and SampleSearch with sampling-based $w$-cutset conditioning (Bidyuk and Dechter, 2007). In $w$-cutset sampling, we sample only the w-cutset variables and then for each sample, we exactly compute the weighted counts of the graphical models using bucket elimination. Using the Rao-Blackwell theorem (Casella and Robert, 1996; Liu, 2001), it is easy to show that $w$-cutset sampling reduces variance. Formally, given a graphical model $\mathcal{G}=\langle\mathbf{X}, \mathbf{D}, \mathbf{F}\rangle$, a $w$-cutset $\mathbf{K}$ and a sample k generated from a proposal distribution $Q(\mathbf{K})$, in $w$-cutset sampling, the weight of

\footnotetext{
${ }^{3}$ Note that we can do this without constructing the messages explicitly.
} 
$\mathbf{k}$ is given by:

$$
w_{w c}(\mathbf{k})=\frac{\sum_{\mathbf{r} \in \mathbf{R}} \prod_{j=1}^{m} F_{j}(\mathbf{r}, \mathbf{K}=\mathbf{k})}{Q(\mathbf{k})}
$$

where $\mathbf{R}=\mathbf{X} \backslash \mathbf{K}$.

It was demonstrated that the higher the $w$-bound (Bidyuk and Dechter, 2007), the smaller the sampling variance. Here also, we select the maximum $w$ such that the resulting bucket elimination algorithm uses less than $512 \mathrm{MB}$ of space. We can choose the appropriate $w$ by using a similar iterative scheme to the one described above for choosing the $i$-bound of IJGP.

4. Markov-LB with SampleCount. SampleCount (Gomes et al., 2007) is an algorithm for estimating the number of solutions of a Boolean Satisfiability problem. It is based on the ApproxCount algorithm of (Wei and Selman, 2005). ApproxCount is based on the formal result of (Valiant, 1987), which states that if one can sample uniformly (or close to it) from the set of solutions of a SAT formula $F$, then one can exactly count (or approximate with a good estimate) the number of solutions of $F$. Consider a SAT formula $F$ with $S$ solutions. If we are able to sample solutions uniformly, then we can compute exactly the fraction of the number of solutions, denoted by $\gamma$ that have a variable $X$ set to True or 1 (and similarly to False or 0 ). If $\gamma$ is greater than zero, we can set $X$ to 1 and simplify $F$ to $F^{\prime}$. The estimate of the number of solutions is now equal to the product of $\frac{1}{\gamma}$ and the number of solutions of $F^{\prime}$. Then, we recursively repeat the process, leading to a series of multipliers, until all variables are assigned a value or until the conditioned formula is easy for exact model counters like Cachet (Sang et al., 2005). To reduce the variance, (Wei and Selman, 2005 ) suggest to set the selected variable to a value that occurs more often in the given set of sampled solutions. In this scheme, the fraction for each variable branching is selected via a solution sampling method called SampleSat (Wei et al., 2004), which is an extension of the well-known local search SAT solver Walksat (Selman et al., 1994).

SampleCount (Gomes et al., 2007) differs from ApproxCount in the following two ways: (a) SampleCount heuristically reduces the variance by branching on variables which are more balanced i.e. variables having multipliers $1 / \gamma$ close to 2 and (b) At each branch point, SampleCount assigns a value to a variable by sampling it with probability 0.5 yielding an unbiased estimate of the solution counts. SampleCount is an importance sampling technique in which the weight of each sample equals $2^{k} \times s$, where $k$ is the number of variables sampled and $s$ is the model count of the SAT formula conditioned on the sampled assignment to the $k$ sampled variables. Therefore, it can be easily combined with Markov-LB yielding the Markov-LB with SampleCount scheme.

In our experiments, we used an implementation of SampleCount available from the authors of (Gomes et al., 2007). Following the recommendations made in (Gomes et al., 2007), we use the following parameters for ApproxCount and SampleCount: (a) Number of samples for SampleSat $=20$, (b) Number of variables remaining to be assigned a value before running Cachet $=100$ and (c) local search cutoff $\alpha=100 K$.

5. Relsat. Relsat (Bayardo and Pehoushek, 2000) is an exact algorithm for counting the number of solutions of a SAT formula. When Relsat is stopped before termination, it yields a lower bound on the solution count. We used an implementation of Relsat available at http: //www. bayardo.org/resources.html. 
We experimented with four versions of Markov-LB (combined on top of SampleSearch, SampleCount and IJGP-Sampling): (a) Markov-LB as given in Algorithm 1, (b) Markov-LB with the average scheme, (c) Markov-LB with the martingale random permutation scheme and (d) Markov-LB with the martingale order statistics scheme. Note that the maximum scheme is subsumed by the Markov-LB with the martingale order statistics scheme. In all our experiments, we set $\alpha=0.99$, namely there is better than $99 \%$ chance that our lower bounds are correct.

\subsubsection{Evaluation Criteria}

We evaluate the performance using the log relative error between the exact value of probability of evidence (or the solution counts for satisfiability problems) and the lower bound generated by the respective techniques. Formally, if $Z$ is the actual probability of evidence (or solution counts) and $\bar{Z}$ is the approximate probability of evidence (or solution counts), the log-relative error denoted by $\Delta$ is given by:

$$
\Delta=\frac{\log (Z)-\log (\bar{Z})}{\log (Z)}
$$

When the exact value of $Z$ is not known, we use the highest lower bound reported by the schemes as a substitute for $Z$ in Equation 33. We use the log relative error because when the probability of evidence is small $\left(\leq 10^{-10}\right)$ or when the solution counts are large (e.g. $\geq 10^{10}$ ) the relative error between the exact and the approximate weighted counts will be arbitrarily close to 1 and we would need a large number of digits to determine the best performing scheme.

Notation in Tables The first column in each table (see for example Table 1) gives the name of the instance. The second column provides raw statistical information about the instance such as: (i) number of variables (n), (ii) average domain size (d), (iii) number of clauses (c) or number of evidence variables (e) and (iv) the upper bound on the treewidth of the instance computed using the min-fill algorithm (w). The third column provides the exact answer for the problem if available while the remaining columns display the output produced by the various schemes after the specified time-bound. The columns Min, Avg, Per and Ord give the log-relative-error $\Delta$ for the minimum, the average, the martingale random permutation and the martingale order statistics schemes respectively. For each instance, the log-relative error of the scheme yielding the best performance is highlighted in bold. The final column Best $L B$ reports the best lower bound.

We organize our results in two parts. We first present results for networks which do not have determinism and compare ATB with IJGP-sampling based Markov-LB schemes. Then, we consider networks which have determinism and compare SampleSearch based Markov-LB with Variable elimination and Conditioning for probabilistic networks and with SampleCount for Boolean satisfiability problems.

\subsection{Results on networks having no determinism}

Table 1 summarizes the results. We ran each algorithm for 2 minutes. We see that our new strategy of Markov-LB scales well with problem size and provides good quality highconfidence lower bounds on most problems. It clearly outperforms the ATB scheme. We discuss the results in detail below. 


\begin{tabular}{|c|c|c|c|c|c|c|c|c|}
\hline \multirow{3}{*}{ Problem } & \multirow[b]{2}{*}{$\langle n, d, e, w\rangle$} & \multirow[b]{2}{*}{ Exact } & \multicolumn{4}{|c|}{$\begin{array}{l}\text { Markov-LB with } \\
\text { IJGP-sampling }\end{array}$} & \multirow[t]{2}{*}{ ATB } & \multirow[b]{2}{*}{ Best } \\
\hline & & & Min & Avg & Per & Ord & & \\
\hline & & $\mathbf{P}(\mathbf{e})$ & $\Delta$ & $\Delta$ & $\Delta$ & $\Delta$ & $\bar{\Delta}$ & LB \\
\hline Alarm & & & & & & & & \\
\hline BN_3 & $\langle 100,2,36\rangle$ & $2.8 \mathrm{E}-13$ & 0.157 & 0.031 & 0.040 & 0.059 & 0.090 & $1.1 \mathrm{E}-13$ \\
\hline BN_4 & $\langle 100,2,51\rangle$ & $3.6 \mathrm{E}-18$ & 0.119 & 0.023 & 0.040 & 0.045 & 0.025 & $1.4 \mathrm{E}-18$ \\
\hline BN_5 & $\langle 125,2,55\rangle$ & $1.8 \mathrm{E}-19$ & 0.095 & 0.020 & 0.021 & 0.030 & 0.069 & $7.7 \mathrm{E}-20$ \\
\hline BN_6 & $\langle 125,2,71\rangle$ & $4.3 \mathrm{E}-26$ & 0.124 & 0.016 & 0.024 & 0.030 & 0.047 & $1.6 \mathrm{E}-26$ \\
\hline BN_11 & $\langle 125,2,46\rangle$ & $8.0 \mathrm{E}-18$ & 0.185 & 0.023 & 0.061 & 0.064 & 0.102 & $3.3 \mathrm{E}-18$ \\
\hline CPCS & & & & & & & & \\
\hline CPCS & $\langle 3$ & $1.3 \mathrm{E}$ & 0.012 & 0.012 & 0.000 & 0.001 & 0.002 & $1.3 \mathrm{E}-25$ \\
\hline CPCS-360-2 & $\langle 360,2,30\rangle$ & $7.6 \mathrm{E}-22$ & 0.045 & 0.015 & 0.010 & 0.010 & 0.000 & $7.6 \mathrm{E}-22$ \\
\hline CPCS-360-3 & $\langle 360,2,40\rangle$ & $1.2 \mathrm{E}-33$ & 0.010 & 0.009 & 0.000 & 0.000 & 0.000 & $1.2 \mathrm{E}-33$ \\
\hline CPCS-360-4 & $\langle 360,2,50\rangle$ & $3.4 \mathrm{E}-38$ & 0.022 & 0.009 & 0.002 & 0.000 & 0.000 & $3.4 \mathrm{E}-38$ \\
\hline CPCS-422-1 & $\langle 422,2,20\rangle$ & $7.2 \mathrm{E}-21 \mid$ & 0.028 & 0.016 & 0.001 & 0.001 & 0.002 & $6.8 \mathrm{E}-21$ \\
\hline CPCS-422-2 & $\langle 422,2,30\rangle$ & $2.7 \mathrm{E}-57$ & 0.005 & 0.005 & 0.000 & 0.000 & 0.000 & $2.7 \mathrm{E}-57$ \\
\hline CPCS-422-3 & $\langle 422,2,40\rangle$ & $6.9 \mathrm{E}-87$ & 0.003 & 0.003 & 0.000 & 0.000 & 0.001 & $6.9 \mathrm{E}-87$ \\
\hline CPCS-422-4 & $\langle 422,2,50\rangle$ & $1.4 \mathrm{E}-73$ & 0.007 & 0.004 & 0.000 & 0.000 & 0.001 & $1.3 \mathrm{E}-73$ \\
\hline Random & & & & & & & & \\
\hline BN_94 & $\langle 53,50,6\rangle$ & $4.0 \mathrm{E}-11$ & 0.235 & 0.029 & 0.063 & 0.025 & 0.028 & $2.2 \mathrm{E}-11$ \\
\hline BN_96 & $\langle 54,50,5\rangle$ & 2.1E-09 & 0.408 & 0.036 & 0.095 & 0.013 & 0.131 & 1.6E-09 \\
\hline BN_98 & $\langle 57,50,6\rangle$ & $1.9 \mathrm{E}-11$ & 0.131 & 0.024 & 0.013 & 0.024 & 0.147 & $1.4 \mathrm{E}-11$ \\
\hline BN_100 & $\langle 58,50,8\rangle$ & $1.6 \mathrm{E}-14$ & 0.521 & 0.022 & 0.079 & 0.041 & 0.134 & $8.1 \mathrm{E}-15$ \\
\hline BN_102 & $\langle\langle 76,50,15\rangle$ & $1.5 \mathrm{E}-26$ & 0.039 & 0.007 & 0.007 & 0.012 & 0.056 & $9.4 \mathrm{E}-27$ \\
\hline
\end{tabular}

Table 1: Table showing the log-relative error $\Delta$ of ATB and four versions of Markov-LB combined with IJGP-sampling for Bayesian networks having no determinism after 2 minutes of CPU time. 
Non-deterministic Alarm networks. The Alarm networks are one of the earliest Bayesian networks designed by medical experts for monitoring patients in intensive care. The evidence in these networks was set at random. These networks have between 100-125 binary nodes. We can see that Markov-LB with IJGP-sampling is slightly superior to ATB accuracy-wise. Among the different versions of Markov-LB with IJGP-sampling, the average scheme performs better than the martingale schemes. The minimum scheme is the worst performing scheme.

The CPCS networks. The CPCS networks are derived from the Computer-based Patient Case Simulation system (Pradhan et al., 1994). The nodes of CPCS networks correspond to diseases and findings and conditional probabilities describe their correlations. The CPCS360b and CPCS422b networks have 360 and 422 variables respectively. We report results on the two networks with $20,30,40$ and 50 randomly selected evidence nodes. We see that the lower bounds reported by ATB are slightly better than Markov-LB with IJGP-sampling on the CPCS360b networks. However, on the CPCS422b networks, MarkovLB with IJGP-sampling gives higher lower bounds. The martingale schemes (the random permutation and the order statistics) give higher lower bounds than the average scheme. Again, the minimum scheme is the weakest.

Random networks. The random networks are randomly generated graphs available from the UAI 2006 evaluation web site. The evidence nodes are generated at random. The networks have between 53 and 76 nodes and the maximum domain size is 50 . We see that Markov-LB is better than ATB on all random networks. The random permutation and the order statistics martingale schemes are slightly better than the average scheme on most instances.

\subsection{Results on networks having determinism}

In this subsection, we report on experiments for networks which have determinism. We experimented with five benchmark domains: (a) Latin square instances, (b) Langford instances, (c) FPGA routing instances, (d) Linkage instances and (e) Relational instances. The task of interest on the first three domains is counting solutions while the task of interest on the remaining domains is computing the probability of evidence.

\subsubsection{Results on Satisfiability model counting}

For model counting, we evaluate the lower bounding power of Markov-LB with SampleSearch and Markov-LB with SampleCount (Gomes et al., 2007). We ran both algorithms for 10 hours on each instance.

Results on the Latin Square instances Our first set of benchmark instances come from the normalized Latin squares domain. A Latin square of order $s$ is an $s \times s$ table filled with $s$ numbers from $\{1, \ldots, s\}$ in such a way that each number occurs exactly once in each row and exactly once in each column. In a normalized Latin square the first row and column are fixed. The task here is to count the number of normalized Latin squares of a given order. The Latin squares were modeled as SAT formulas using the extended encoding given in (Gomes and Shmoys, 2002). The exact counts for these formulas are known up to order 11 (Ritter, 2003). 


\begin{tabular}{|c|c|c|c|c|c|c|c|c|c|c|c|c|}
\hline \multirow{3}{*}{ Problem } & \multirow[b]{2}{*}{$\langle n, k, c, w\rangle$} & \multirow[b]{2}{*}{ Exact } & \multicolumn{4}{|c|}{$\begin{array}{l}\text { Markov-LB with } \\
\text { SampleSearch }\end{array}$} & \multicolumn{4}{|c|}{$\begin{array}{l}\text { Markov-LB with } \\
\text { SampleCount }\end{array}$} & \multirow[t]{2}{*}{$\begin{array}{l}\text { REL } \\
\text { SAT }\end{array}$} & \multirow[b]{2}{*}{ Best } \\
\hline & & & Min & Avg & Per & Ord & Min & Avg & Per & Ord & & \\
\hline & & & $\Delta$ & $\Delta$ & $\Delta$ & $\Delta$ & $\Delta$ & $\Delta$ & $\Delta$ & $\Delta$ & $\Delta$ & LB \\
\hline \begin{tabular}{|l|} 
ls8-norm \\
\end{tabular} & $\langle 512,2,5584,255\rangle$ & $.40 \mathrm{E}+11$ & 0.387 & 0.012 & 0.068 & 0.095 & 0.310 & 0.027 & 0.090 & 0.090 & 0.344 & $88 \mathrm{E}+11$ \\
\hline \begin{tabular}{|l|} 
ls9-norm \\
\end{tabular} & $\langle 729,2,9009,363\rangle$ & $3.80 \mathrm{E}+17$ & 0.347 & 0.021 & 0.055 & 0.070 & 0.294 & 0.030 & 0.097 & 0.074 & 0.579 & $1.59 \mathrm{E}+17$ \\
\hline ls10-norm & $\langle\langle 1000,2,13820,676\rangle$ & $7.60 \mathrm{E}+24$ & 0.304 & 0.002 & 0.077 & 0.044 & 0.237 & 0.016 & 0.054 & 0.050 & 0.710 & $6.93 \mathrm{E}+24$ \\
\hline ls11-norm & $\langle\langle 1331,2,20350,956\rangle$ & $5.40 \mathrm{E}+33$ & 0.287 & 0.023 & 0.102 & 0.026 & 0.227 & 0.036 & 0.094 & 0.034 & 0.783 & $7.37 \mathrm{E}+34$ \\
\hline ls12-norm & $\langle\langle 1728,2,28968,1044\rangle$ & & 0.251 & 0.007 & 0.045 & 0.011 & 0.232 & 0.000 & 0.079 & 0.002 & 0.833 & $3.23 \mathrm{E}+43$ \\
\hline ls13-norm & $\langle\langle 2197,2,40079,1558\rangle$ & & 0.250 & 0.005 & 0.080 & 0.000 & 0.194 & 0.015 & 0.087 & 0.044 & 0.870 & $1.26 \mathrm{E}+55$ \\
\hline 1s14-norm & $\langle\langle 2744,2,54124,1971\rangle$ & & 0.174 & 0.010 & 0.057 & 0.000 & 0.140 & 0.043 & 0.065 & 0.026 & 0.899 & $2.72 \mathrm{E}+67$ \\
\hline ls15-norm & $\langle\langle 3375,2,71580,2523\rangle$ & & 0.189 & 0.015 & 0.080 & 0.000 & 0.130 & 0.053 & 0.077 & 0.062 & 0.923 & $4.84 \mathrm{E}+82$ \\
\hline ls16-norm & $\langle 4096,2,92960,2758\rangle$ & & 0.158 & 0.000 & 0.055 & 0.001 & 0.108 & 0.030 & 0.053 & 0.007 & $\mathrm{X}$ & $1.16 \mathrm{E}+97$ \\
\hline
\end{tabular}

Table 2: Table showing the log-relative error $\Delta$ of Relsat and four versions of MarkovLB combined with SampleSearch and SampleCount respectively for Latin Square instances after 10 hours of CPU time.

\begin{tabular}{|c|c|c|c|c|c|c|c|c|c|c|c|c|}
\hline \multirow{3}{*}{ Problem } & \multirow[b]{2}{*}{$\langle n, k, c, w\rangle$} & \multirow{2}{*}{$\begin{array}{l}\text { Ex } \\
\text { act }\end{array}$} & \multicolumn{4}{|c|}{$\begin{array}{l}\text { Markov-LB with } \\
\text { SampleSearch }\end{array}$} & \multicolumn{4}{|c|}{$\begin{array}{l}\text { Markov-LB with } \\
\text { SampleCount }\end{array}$} & \multirow[t]{2}{*}{$\begin{array}{l}\text { REL } \\
\text { SAT }\end{array}$} & \multirow[b]{2}{*}{ Best } \\
\hline & & & Min & Avg & Per & Ord & Min & Avg & Per & Ord & & \\
\hline & & & $\Delta$ & $\Delta$ & $\Delta$ & $\Delta$ & $\Delta$ & $\Delta$ & $\Delta$ & $\Delta$ & $\Delta$ & LB \\
\hline lang12 & $\langle 576,2,13584,383\rangle$ & $2.16 \mathrm{E}+05$ & 0.464 & 0.051 & 0.128 & 0.171 & 0.455 & 0.067 & 0.103 & 0.175 & 0.000 & $2.16 \mathrm{E}+05$ \\
\hline lang16 & $\langle 1024,2,32320,639\rangle$ & $6.53 \mathrm{E}+08$ & 0.475 & 0.008 & 0.106 & 0.131 & 0.378 & 0.019 & 0.097 & 0.023 & 0.365 & $7.68 \mathrm{E}+08$ \\
\hline lang19 & $\langle 1444,2,54226,927\rangle$ & $5.13 \mathrm{E}+11$ & 0.405 & $\overline{0.041}$ & 0.109 & 0.095 & 0.420 & 0.156 & 0.219 & 0.200 & 0.636 & $1.70 \mathrm{E}+11$ \\
\hline lang20 & $\langle 1600,2,63280,1023\rangle$ & $5.27 \mathrm{E}+12$ & 0.411 & 0.031 & 0.150 & 0.102 & 0.424 & 0.217 & \begin{tabular}{|l|l|}
0.188 \\
\end{tabular} & 0.123 & 0.685 & $2.13 \mathrm{E}+12$ \\
\hline lang23 & $\langle 2116,2,9$ & $7.60 \mathrm{E}+15$ & 0.389 & $\overline{0.058}$ & 0.119 & 0.100 & 0.418 & 0.215 & 0.284 & 0.211 & $\mathrm{X}$ & $9.15 \mathrm{E}+14$ \\
\hline lang24 & $\langle 2304,2,10$ & $9.37 \mathrm{E}+16$ & 0.258 & 0.076 & 0.043 & 0.05 & ] .28. & 0.220 & 0.203 & 0.220 & $\bar{X}$ & $1.74 \mathrm{E}+16$ \\
\hline lang27 & $\langle 2916,2,156114,1919\rangle$ & & 0.26 & 0.000 & 0.093 & 0.107 & 0.364 & 0.264 & 0.291 & 0.267 & $\mathrm{X}$ & $7.67 \mathrm{E}+19$ \\
\hline
\end{tabular}

Table 3: Table showing the log-relative error $\Delta$ of Relsat and four versions of Markov-LB combined with SampleSearch and SampleCount respectively for Langford instances after 10 hours of CPU time.

Table 2 shows the results. The exact counts for Latin square instances are known only up to order 11. As pointed out earlier, when the exact results are not known, we use the highest lower bound reported by the schemes as a substitute for $Z$ in Equation 33 .

Among the different versions of Markov-LB with SampleSearch, we see that the average scheme performs better than the martingale order statistics scheme on 5 out of 8 instances while the martingale order statistics scheme is superior on the other 3 instances. The minimum scheme is the weakest scheme while the martingale random permutation scheme is between the minimum scheme and the average and martingale order statistics scheme.

Among the different versions of Markov-LB with SampleCount, we see very similar performance. SampleSearch with Markov-LB generates better lower bounds than SampleCount with Markov-LB on 6 out of the 8 instances. The lower bounds output by Relsat are several orders of magnitude lower than those output by Markov-LB with SampleSearch and Markov-LB with SampleCount.

Results on Langford instances Our second set of benchmark instances come from the Langford's problem domain. The problem is parameterized by its (integer) size denoted by $s$. Given a set of $s$ numbers $\{1,2, \ldots, s\}$, the problem is to produce a sequence of length $2 s$ such that each $i \in\{1,2, \ldots, s\}$ appears twice in the sequence and the two occurrences of $i$ are exactly $i$ apart from each other. This problem is satisfiable only if $n$ is 0 or 3 modulo 4. We encoded the Langford problem as a SAT formula using the channeling SAT encoding described in (Walsh, 2001). 


\begin{tabular}{|c|c|c|c|c|c|c|c|c|c|c|c|c|}
\hline \multirow{3}{*}{ Problem } & \multirow{3}{*}{$\langle n, k, c, w\rangle$} & \multirow{2}{*}{$\begin{array}{l}\text { Ex- } \\
\text { act }\end{array}$} & \multicolumn{4}{|c|}{$\begin{array}{l}\text { Markov-LB with } \\
\text { SampleSearch }\end{array}$} & \multicolumn{4}{|c|}{$\begin{array}{l}\text { Markov-LB with } \\
\text { SampleCount }\end{array}$} & \multirow[t]{2}{*}{$\begin{array}{l}\text { REL } \\
\text { SAT }\end{array}$} & \multirow[b]{2}{*}{ Best } \\
\hline & & & Min & Avg & Per & Ord & Min & Avg & Per & Ord & & \\
\hline & & & $\Delta$ & $\Delta$ & $\Delta$ & $\Delta$ & $\Delta$ & $\Delta$ & $\Delta$ & $\Delta$ & $\Delta$ & LB \\
\hline 9symml_gr_2pin_w6 & $\langle 2604,2,36994,413\rangle$ & & 0.192 & 0.000 & 0.075 & 0.006 & 0.087 & 0.073 & 0.076 & 0.075 & 0.491 & $2.76 \mathrm{E}+53$ \\
\hline 9symml_gr_rcs_w6 & $\langle 1554,2,29119,613\rangle$ & & 0.237 & 0.016 & 0.117 & 0.023 & 0.117 & 0.060 & 0.041 & 0.009 & 0.000 & $9.95 \mathrm{E}+84$ \\
\hline alu2_gr_rcs_w8 & $\langle 4080,2,83902,1470\rangle$ & & 0.224 & 0.097 & 0.152 & 0.102 & 0.000 & 0.906 & 0.023 & 0.345 & 0.762 & $1.47 \mathrm{E}+235$ \\
\hline apex7_gr_2pin_w5 & $\langle 1983,2,15358,188\rangle$ & & 0.158 & 0.003 & 0.073 & 0.000 & 0.064 & 0.023 & 0.047 & 0.036 & 0.547 & $2.71 \mathrm{E}+93$ \\
\hline apex7_gr_rcs_w5 & $\langle 1500,2,11695,290\rangle$ & & 0.228 & 0.037 & 0.118 & 0.038 & 0.099 & 0.000 & 0.028 & 0.008 & 0.670 & $3.04 \mathrm{E}+139$ \\
\hline c499_gr_2pin_w6 & $\langle 2070,2,22470,263\rangle$ & & 0.262 & 0.012 & 0.092 & 0.000 & $\mathrm{X}$ & $\mathrm{X}$ & $\mathrm{X}$ & $\mathrm{X}$ & 0.376 & $6.84 \mathrm{E}+54$ \\
\hline c499_gr_rcs_w6 & $\langle 1872,2,18870,462\rangle$ & & 0.310 & 0.046 & 0.164 & 0.043 & 0.083 & 0.042 & 0.062 & 0.000 & 0.391 & $1.07 \mathrm{E}+88$ \\
\hline c880_gr_rcs_w7 & $\langle\langle 4592,2,61745,1024\rangle$ & & 0.223 & 0.110 & 0.142 & 0.110 & 0.000 & 0.000 & 0.000 & 0.003 & 0.845 & $1.37 \mathrm{E}+278$ \\
\hline example2_gr_2pin_w6 & $\langle 3603,2,41023,350\rangle$ & & 0.112 & 0.000 & 0.026 & 0.000 & 0.005 & 0.005 & 0.005 & 0.005 & 0.756 & $2.78 \mathrm{E}+159$ \\
\hline example2_gr_rcs_w6 & $\langle 2664,2,27684,476\rangle$ & & 0.176 & 0.050 & 0.079 & 0.054 & 0.056 & 0.005 & 0.000 & 0.005 & 0.722 & $1.47 \mathrm{E}+263$ \\
\hline term1_gr_2pin_w4 & $\langle 746,2,3964,31\rangle$ & & 0.199 & 0.000 & 0.077 & 0.002 & $\mathrm{X}$ & $\mathrm{X}$ & $\mathrm{X}$ & $\mathrm{X}$ & 0.141 & $7.68 \mathrm{E}+39$ \\
\hline term1_gr_rcs_w4 & $\langle 808,2,3290,57\rangle$ & & 0.252 & 0.000 & 0.090 & 0.017 & $\mathrm{X}$ & $\mathrm{X}$ & $\mathrm{X}$ & $\mathrm{X}$ & 0.175 & $4.97 \mathrm{E}+55$ \\
\hline too_large_gr_rcs_w7 & $\langle 3633,2,50373,1069\rangle$ & & 0.156 & 0.026 & 0.073 & 0.000 & $\mathrm{X}$ & $\mathrm{X}$ & $\mathrm{X}$ & $\mathrm{X}$ & 0.608 & $7.73 \mathrm{E}+182$ \\
\hline too_large_gr_rcs_w8 & $\langle\langle 4152,2,57495,1330\rangle$ & & 0.147 & 0.000 & 0.038 & 0.020 & $\mathrm{X}$ & $\mathrm{X}$ & $\mathrm{X}$ & $\mathrm{X}$ & 0.750 & $8.36 \mathrm{E}+246$ \\
\hline vda_gr_rcs_w9 & $\langle 6498,2,130997,2402\rangle$ & & 0.088 & 0.009 & 0.030 & 0.000 & $\mathrm{X}$ & $\mathrm{X}$ & $\mathrm{X}$ & $\mathrm{X}$ & 0.749 & $5.04 \mathrm{E}+300$ \\
\hline
\end{tabular}

Table 4: Table showing the log-relative error $\Delta$ of Relsat and four versions of Markov-LB combined with SampleSearch and SampleCount respectively for FPGA routing instances after 10 hours of CPU time.

Table 3 shows the results. Among the different versions of Markov-LB with SampleSearch, we see again the superiority of the average scheme. The martingale order statistics and random permutation schemes are the second and the third best respectively. Among the different versions of SampleCount based Markov-LB, we see a similar trend where the average scheme performs better than other schemes on 6 out of the 7 instances.

Markov-LB with SampleSearch outperforms Markov-LB with SampleCount on 6 out of the 7 instances. The lower bounds output by Relsat are inferior by several orders of magnitude to the Markov-LB based lower bounds except on the lang12 instance which RESLAT solves exactly.

Results on FPGA routing instances Our final SAT domain is that of the FPGA routing instances. These instances are constructed by reducing FPGA (Field Programmable Gate Array) detailed routing problems into a satisfiability formula. The instances were generated by Gi-Joon Nam and were used in the SAT 2002 competition (Simon et al., 2005).

We see (Table 4) a similar behavior to the Langford and Latin square instances in that the average and the martingale order statistics schemes are better than other schemes. SampleSearch based Markov-LB yields better lower bounds than SampleCount based MarkovLB on 11 out of the 17 instances. As in the other benchmarks, the lower bounds output by Relsat are inferior by several orders of magnitude.

\subsubsection{Results on Linkage instances}

The Linkage networks are generated by converting biological linkage analysis data into a Bayesian or Markov network. Linkage analysis is a statistical method for mapping genes onto a chromosome (Ott, 1999). This is very useful in practice for identifying disease genes. The input is an ordered list of loci $L_{1}, \ldots, L_{k+1}$ with allele frequencies at each locus and a pedigree with some individuals typed at some loci. The goal of linkage analysis is to evaluate the likelihood of a candidate vector $\left[\theta_{1}, \ldots, \theta_{k}\right]$ of recombination fractions for the 


\begin{tabular}{|c|c|c|c|c|c|c|c|c|}
\hline \multirow{3}{*}{ Problem } & \multirow[b]{2}{*}{$\langle n, k, c, w\rangle$} & \multirow[b]{2}{*}{ Exact } & \multicolumn{4}{|c|}{$\begin{array}{l}\text { Markov-LB with } \\
\text { SampleSearch }\end{array}$} & \multirow[t]{2}{*}{ VEC } & \multirow{2}{*}{$\begin{array}{c}\text { Best } \\
\text { LB }\end{array}$} \\
\hline & & & Min & Avg & Per & Ord & & \\
\hline & & & $\Delta$ & $\Delta$ & $\Delta$ & $\Delta$ & $\Delta$ & \\
\hline BN_69.uai & $\langle 777,7,78,47\rangle$ & $5.28 \mathrm{E}-54$ & 0.082 & 0.029 & 0.031 & 0.034 & 0.140 & $1.56 \mathrm{E}-55$ \\
\hline BN_70.uai & $\langle 2315,5,159,87\rangle$ & $2.00 \mathrm{E}-71$ & 0.275 & 0.035 & 0.101 & 0.046 & 0.147 & $6.24 \mathrm{E}-74$ \\
\hline BN_71.uai & $\langle 1740,6,202,70\rangle$ & $5.12 \mathrm{E}-111$ & 0.052 & 0.009 & 0.019 & 0.017 & 0.035 & $5.76 \mathrm{E}-112$ \\
\hline BN_72.uai & $\langle 2155,6,252,86\rangle$ & $4.21 \mathrm{E}-150$ & 0.021 & 0.002 & 0.004 & 0.007 & 0.023 & $2.38 \mathrm{E}-150$ \\
\hline BN_73.uai & $\langle 2140,5,216,101\rangle$ & $2.26 \mathrm{E}-113$ & 0.172 & 0.020 & 0.059 & 0.026 & 0.121 & $1.19 \mathrm{E}-115$ \\
\hline BN_74.uai & $\langle 749,6,66,45\rangle$ & $3.75 \mathrm{E}-45$ & 0.233 & 0.035 & 0.035 & 0.049 & 0.069 & $1.09 \mathrm{E}-46$ \\
\hline BN_75.uai & $\langle 1820,5,155,92\rangle$ & $5.88 \mathrm{E}-91$ & 0.077 & 0.005 & 0.024 & 0.019 & 0.067 & $1.98 \mathrm{E}-91$ \\
\hline BN_76.uai & $\langle 2155,7,169,64\rangle$ & $4.93 \mathrm{E}-110$ & 0.109 & 0.015 & 0.043 & 0.018 & 0.153 & $1.03 \mathrm{E}-111$ \\
\hline
\end{tabular}

Table 5: Table showing the log-relative error $\Delta$ of VEC and four versions of Markov-LB combined with SampleSearch for Linkage instances from the UAI 2006 evaluation after 3 hours of CPU time.

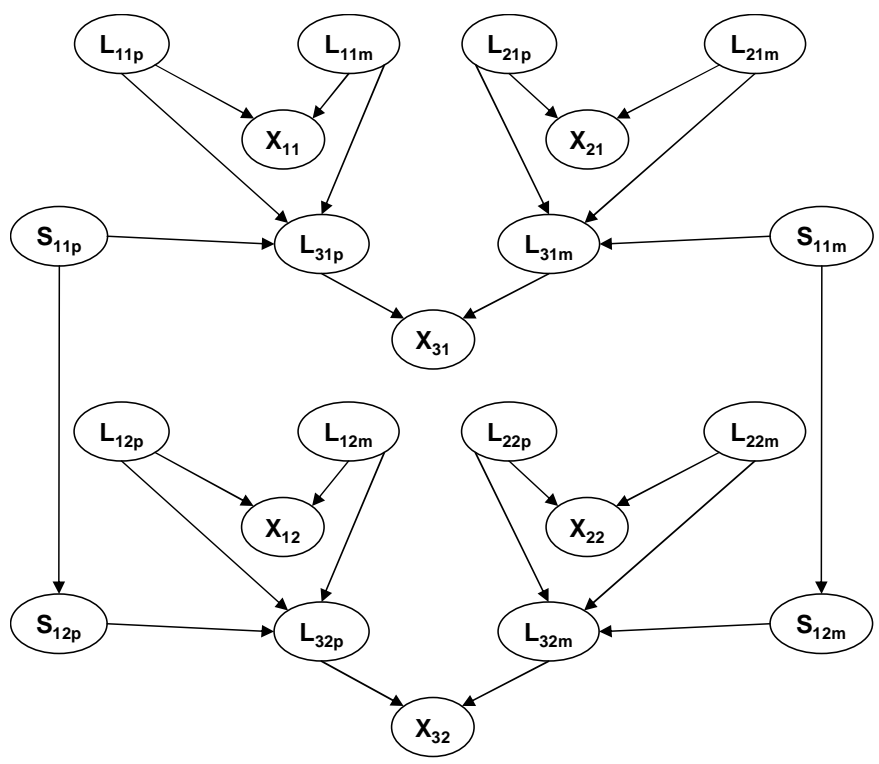

Figure 1: A fragment of a Bayesian network used in genetic linkage analysis.

input pedigree and locus order. The component $\theta_{i}$ is the candidate recombination fraction between the loci $L_{i}$ and $L_{i+1}$.

The pedigree data can be represented as a Bayesian network with three types of random variables: genetic loci variables which represent the genotypes of the individuals in the pedigree (two genetic loci variables per individual per locus, one for the paternal allele and one for the maternal allele), phenotype variables, and selector variables which are auxiliary variables used to represent the gene flow in the pedigree. Figure 1 represents a fragment of a network that describes parents-child interactions in a simple 2-loci analysis. The genetic loci variables of individual $i$ at locus $j$ are denoted by $L_{i, j p}$ and $L_{i, j m}$. Variables $X_{i, j}, S_{i, j p}$ and $S_{i, j m}$ denote the phenotype variable, the paternal selector variable and the maternal selector variable of individual $i$ at locus $j$, respectively. The conditional probability tables that correspond to the selector variables are parameterized by the recombination ratio $\theta$. The remaining tables contain only deterministic information. It can be shown that given the pedigree data, computing the likelihood of the recombination fractions is equivalent to 


\begin{tabular}{|c|c|c|c|c|c|c|c|c|}
\hline \multirow{3}{*}{ Problem } & \multirow[b]{2}{*}{$\langle n, k, c, w\rangle$} & \multirow[b]{2}{*}{ Exact } & \multicolumn{4}{|c|}{$\begin{array}{c}\text { Markov-LB with } \\
\text { SampleSearch }\end{array}$} & \multirow[t]{2}{*}{ VEC } & \multirow{2}{*}{$\begin{array}{c}\text { Best } \\
\text { LB }\end{array}$} \\
\hline & & & Min & Avg & Per & Ord & & \\
\hline & & & $\Delta$ & $\Delta$ & $\Delta$ & $\Delta$ & $\Delta$ & \\
\hline pedigree18.uai & $\langle 1184,1,0,26\rangle$ & $7.18 \mathrm{E}-79$ & 0.062 & 0.004 & 0.011 & 0.016 & 0.000 & \begin{tabular}{|l|}
$7.18 \mathrm{E}-79$ \\
\end{tabular} \\
\hline pedigree1.uai & $\langle 334,2,0,20\rangle$ & $7.81 \mathrm{E}-15$ & 0.034 & 0.020 & 0.020 & 0.020 & 0.000 & $7.81 \mathrm{E}-15$ \\
\hline pedigree20.uai & $\langle 437,2,0,25\rangle$ & $2.34 \mathrm{E}-30$ & 0.208 & 0.010 & 0.011 & 0.029 & 0.000 & $2.34 \mathrm{E}-30$ \\
\hline pedigree23.uai & $\langle 402,1,0,26\rangle$ & $2.78 \mathrm{E}-39$ & 0.093 & 0.007 & 0.016 & 0.019 & 0.000 & $2.78 \mathrm{E}-39$ \\
\hline pedigree25.uai & $\langle 1289,1,0,38\rangle$ & $2.12 \mathrm{E}-119$ & 0.006 & 0.022 & 0.019 & 0.019 & 0.024 & $1.69 \mathrm{E}-116$ \\
\hline pedigree30.uai & $\langle 1289,1,0,27\rangle$ & $4.03 \mathrm{E}-88$ & 0.014 & 0.039 & 0.039 & 0.035 & 0.042 & $1.85 \mathrm{E}-84$ \\
\hline pedigree37.uai & $\langle 1032,1,0,25\rangle$ & $2.63 \mathrm{E}-117$ & 0.031 & 0.005 & 0.005 & 0.006 & 0.000 & $2.63 \mathrm{E}-117$ \\
\hline e38.uai & $\langle 724,1,0,18\rangle$ & $5.64 \mathrm{E}-55$ & 0.197 & 0.010 & 0.024 & 0.023 & 0.000 & 5.65E-55 \\
\hline pedigree39.uai & $\langle 1272,1,0,29\rangle$ & $6.32 \mathrm{E}-103$ & 0.039 & 0.003 & 0.001 & 0.007 & 0.000 & $7.96 \mathrm{E}-103$ \\
\hline pedigree42.uai & $\langle 448,2,0,23\rangle$ & $1.73 \mathrm{E}-31$ & 0.024 & 0.009 & 0.007 & 0.010 & 0.000 & $1.73 \mathrm{E}-31$ \\
\hline pedigree19.uai & $\langle 793,2,0,23\rangle$ & & 0.158 & 0.018 & 0.000 & 0.031 & 0.011 & 3.67E-59 \\
\hline pedigree31.uai & $\langle 1183,2,0,45\rangle$ & & 0.059 & 0.000 & 0.003 & 0.011 & 0.083 & $1.03 \mathrm{E}-70$ \\
\hline pedigree34.uai & $\langle 1160,1,0,59\rangle$ & & 0.211 & 0.006 & 0.000 & 0.012 & 0.174 & $4.34 \mathrm{E}-65$ \\
\hline pedigree13.uai & $\langle 1077,1,0,51\rangle$ & & 0.175 & 0.000 & 0.038 & 0.023 & 0.163 & $2.94 \mathrm{E}-32$ \\
\hline pedigree40.uai & $\langle 1030,2,0,49\rangle$ & & 0.126 & 0.000 & 0.036 & 0.008 & 0.025 & $4.26 \mathrm{E}-89$ \\
\hline pedigree41.uai & $\langle 1062,2,0,52\rangle$ & & 0.079 & 0.000 & 0.012 & 0.010 & 0.049 & $2.29 \mathrm{E}-77$ \\
\hline pedigree44.uai & $\langle 811,1,0,29\rangle$ & & 0.045 & 0.002 & 0.007 & 0.009 & 0.000 & $2.23 \mathrm{E}-64$ \\
\hline pedigree51.uai & $\langle 1152,1,0,51\rangle$ & & 0.150 & 0.003 & 0.027 & 0.000 & 0.139 & $1.01 \mathrm{E}-74$ \\
\hline pedigree7.uai & $\langle 1068,1,0,56\rangle$ & & 0.127 & 0.000 & 0.019 & 0.009 & 0.101 & $6.42 \mathrm{E}-66$ \\
\hline pedigree9.uai & $\langle 1118,2,0,41\rangle$ & & 0.072 & 0.000 & 0.009 & 0.009 & 0.028 & $1.41 \mathrm{E}-79$ \\
\hline
\end{tabular}

Table 6: Table showing the log-relative error $\Delta$ of VEC and four versions of Markov-LB combined with SampleSearch for Linkage instances from the UAI 2008 evaluation after 3 hours of CPU time.

computing the probability of evidence on the Bayesian network that model the problem (for more details consult (Fishelson and Geiger, 2003)).

Table 5 shows the results for linkage instances used in the UAI 2006 evaluation (Bilmes and Dechter, 2006). Here, we compare Markov-LB with SampleSearch with VEC. ATB (Bidyuk and Dechter, 2006) does not work on instances having determinism and therefore we do not report on it here. We clearly see that SampleSearch based Markov-LB yields higher lower bounds than VEC. Remember, however that the lower bounds output by VEC are correct (with probability 1) while the lower bounds output by Markov-LB are correct with probability $\geq 0.99$. We see that the average scheme is the best performing scheme. Martingale order statistics scheme is the second best while the Martingale random permutation scheme is the third best. The minimum scheme is the worst performing scheme.

Table 6 reports the results on Linkage instances encoded as Markov networks, used in the UAI 2008 evaluation (Darwiche et al., 2008). VEC solves 10 instances exactly. On these instances, the lower bound output by SampleSearch based Markov-LB are quite accurate as evidenced by the small log relative error. On instances which VEC does not solve exactly, we clearly see that Markov-LB with SampleSearch yields higher lower bounds than VEC.

Comparing between different versions of Markov-LB, we see that the average scheme is overall the best performing scheme. The Martingale order statistics scheme is the second best scheme while the Martingale random permutation scheme is the third best. 


\begin{tabular}{|c|c|c|c|c|c|c|c|c|}
\hline \multirow{3}{*}{ Problem } & \multirow[b]{2}{*}{$\langle n, k, c, w\rangle$} & \multirow[b]{2}{*}{ Exact } & \multicolumn{4}{|c|}{$\begin{array}{l}\text { Markov-LB with } \\
\text { SampleSearch }\end{array}$} & \multirow[t]{2}{*}{ VEC } & \multirow{2}{*}{$\begin{array}{l}\text { Best } \\
\text { LB }\end{array}$} \\
\hline & & & Min & Avg & Per & Ord & & \\
\hline & & & $\Delta$ & $\Delta$ & $\Delta$ & $\Delta$ & $\Delta$ & \\
\hline fs-01.uai & $\overline{\langle 10,2,7,2\rangle}$ & 00E-01 & 0.000 & 0.000 & 0.000 & 0.000 & 0.000 & $5.00 \mathrm{E}-01$ \\
\hline fs-04.uai & $\langle 262,2,226,12\rangle$ & $1.53 \mathrm{E}-05$ & 0.116 & 0.116 & 0.116 & 0.116 & 0.000 & $1.53 \mathrm{E}-05$ \\
\hline fs-07.uai & $\langle 1225,2,1120,35\rangle$ & $9.80 \mathrm{E}-17$ & 0.028 & 0.004 & 0.014 & 0.016 & 0.079 & $1.78 \mathrm{E}-15$ \\
\hline fs-10.uai & $\langle 3385,2,3175,71\rangle$ & $7.89 \mathrm{E}-31$ & 0.071 & 0.064 & 0.064 & 0.065 & $\mathrm{X}$ & $9.57 \mathrm{E}-33$ \\
\hline fs-13.uai & $\langle 7228,2,6877,119\rangle$ & $1.34 \mathrm{E}-51$ & 0.077 & 0.077 & 0.077 & 0.077 & $\overline{\mathrm{X}}$ & $1.69 \mathrm{E}-55$ \\
\hline fs-16.1 & $\langle 13240,2,12712,171\rangle$ & $8.64 \mathrm{E}-78$ & 0.085 & 0.019 & 0.048 & 0.025 & $\bar{X}$ & $3.04 \mathrm{E}-79$ \\
\hline fs-19.uai & $\langle 21907,2,21166,243\rangle$ & $2.13 \mathrm{E}-109$ & 0.051 & 0.050 & 0.050 & 0.050 & $\mathrm{X}$ & $8.40 \mathrm{E}-115$ \\
\hline fs-22.uai & $\langle 33715,2,32725,335\rangle$ & $2.00 \mathrm{E}-146$ & 0.053 & 0.006 & 0.022 & 0.009 & $\overline{\mathrm{X}}$ & $2.51 \mathrm{E}-147$ \\
\hline fs-25.uai & $\langle 49150,2,47875,431\rangle$ & $7.18 \mathrm{E}-189$ & 0.050 & 0.005 & 0.026 & 0.004 & $\mathrm{X}$ & $1.57 \mathrm{E}-189$ \\
\hline fs-28.uai & $\langle 68698,2,67102,527\rangle$ & $9.83 \mathrm{E}-237$ & 0.231 & 0.017 & 0.023 & 0.011 & $\mathrm{X}$ & $4.53 \mathrm{E}-237$ \\
\hline fs-29.uai & $\langle 76212,2,74501,559\rangle$ & $6.82 \mathrm{E}-254$ & 0.259 & 0.101 & 0.201 & 0.027 & $\mathrm{X}$ & $9.44 \mathrm{E}-255$ \\
\hline mastermind_03_08_03 & $\langle 1220,2,48,20\rangle$ & 9.79E-08 & 0.283 & 0.039 & 0.034 & 0.096 & 0.000 & 9.79E-08 \\
\hline mastermind_03_08_04 & $\langle 2288,2,64,30\rangle$ & $8.77 \mathrm{E}-09$ & 0.562 & 0.045 & 0.145 & 0.131 & 0.000 & $8.77 \mathrm{E}-09$ \\
\hline mastermind_03_08_05 & $\langle 3692,2,80,42\rangle$ & $8.90 \mathrm{E}-11$ & 0.432 & 0.041 & 0.021 & 0.095 & 0.000 & $1.44 \mathrm{E}-10$ \\
\hline mastermind_04_08_03 & $\langle 1418,2,48,22\rangle$ & $8.39 \mathrm{E}-08$ & 0.297 & 0.041 & 0.072 & 0.082 & 0.000 & $8.39 \mathrm{E}-08$ \\
\hline mastermind_04_08_04 & $\langle 2616,2,64,33\rangle$ & $2.20 \mathrm{E}-08$ & 0.640 & 0.026 & 0.155 & 0.103 & 0.034 & $1.38 \mathrm{E}-08$ \\
\hline mastermind_05_08_03 & $\langle 1616,2,48,28\rangle$ & $5.30 \mathrm{E}-07$ & 0.625 & 0.062 & 0.188 & 0.185 & 0.000 & $5.30 \mathrm{E}-07$ \\
\hline mastermind_06_08_03 & $\langle 1814,2,48,31\rangle$ & $1.80 \mathrm{E}-08$ & 0.510 & 0.058 & 0.193 & 0.175 & 0.000 & $1.80 \mathrm{E}-08$ \\
\hline mastermind_10_08_03 & $\langle 2606,2,48,56\rangle$ & $1.92 \mathrm{E}-07$ & 0.839 & 0.058 & 0.297 & 0.162 & 0.058 & $7.90 \mathrm{E}-08$ \\
\hline
\end{tabular}

Table 7: Table showing the log-relative error $\Delta$ of VEC and four versions of Markov-LB combined with SampleSearch for relational instances after 3 hours of CPU time.

\subsubsection{Results on Relational instances}

The relational instances are generated by grounding the relational Bayesian networks using the Primula tool (Chavira et al., 2006). We experimented with ten Friends and Smoker networks and six mastermind networks from this domain which have between 262 to 76,212 variables.

In Table 7, we report the results on instances with 10 Friends and Smoker networks and 6 mastermind networks from this domain which have between 262 to 76,212 variables. On the 11 friends and smokers network, we can see that as the problems get larger the lower bounds output by Markov-LB with SampleSearch are higher than VEC. This clearly indicates that Markov-LB with SampleSearch is more scalable than VEC. VEC solves exactly six out of the eight mastermind instances while on the remaining two instances Markov-LB with SampleSearch yields higher lower bounds than VEC.

\subsection{Summary of Experimental Results}

Based on our large scale experimental evaluation, we see that applying Markov inequality and its generalizations to multiple samples generated by SampleSearch and IJGP-Sampling is more scalable than deterministic approaches such as Variable elimination and conditioning (VEC), Relsat and ATB. Among the different versions of Markov-LB, we find that the average and martingale order statistics schemes consistently yield higher lower bounds and therefore they should be preferred over the minimum scheme as well as the martingale random permutation scheme. 


\section{Conclusion and Future Work}

Weighted counting is an important problem in the context of graphical models because it includes many problems such as finding the probability of evidence and the partition function as special cases. In this paper, we considered the problem of probabilistically lower bounding weighted counts and introduced several schemes based on importance sampling and Markov inequality for it. Our new schemes extend the one-sample Markov inequality to multiple samples using various statistics such as the average, maximum and order, which significantly improves its power and accuracy. Empirically, we showed that our new schemes combined on top of state of-the-art importance sampling approaches such as IJGP-sampling (Gogate, 2009), SampleSearch (Gogate and Dechter, 2011), and SampleCount (Gomes et al., 2007) are substantially superior to state-of-the-art deterministic algorithms such as ATB (Bidyuk et al., 2010), Relsat (Bayardo and Pehoushek, 2000) and variable elimination and conditioning.

Several avenues remain for future work. An interesting future work is to develop randomized algorithms for probabilistically upper bounding the weighted counts. Another line of future work is relaxing the probabilistic guarantees requirement to a weaker statistical guarantees requirement. Some initial results on this are reported in (Kroc et al., 2008; Davies and Bacchus, 2008). A third line of future work is combining various schemes. A possible approach is to divide the samples into $k$ bins, apply different schemes to different bins, consider the output as $k$ samples and then apply one of the five schemes on the $k$ samples.

\section{References}

Allen, D. and Darwiche, A. (2008). Rc_link: Genetic linkage analysis using Bayesian networks. International Journal of Approximate Reasoning, 48(2):499-525.

Bayardo, R. J. and Pehoushek, J. D. (2000). Counting models using connected components. In Proceedings of Seventeenth National Conference on Artificial Intelligence, pages 157162.

Bergeron, J. (2000). Writing Testbenches: Functional Verification of HDL Models. Kluwer Academic Publishers.

Bidyuk, B. and Dechter, R. (2006). An anytime scheme for bounding posterior beliefs. In Proceedings of the Twenty-First AAAI Conference on Artificial Intelligence, pages 1095-1100.

Bidyuk, B. and Dechter, R. (2007). Cutset sampling for Bayesian networks. Journal of Artificial Intelligence Research, 28:1-48.

Bidyuk, B., Dechter, R., and Rollon, E. (2010). Active tuples-based scheme for bounding posterior beliefs. Journal of Artificial Intelligence Research, 39:335-371.

Bilmes, J. and Dechter, R. (2006). Evaluation of Probabilistic Inference Systems of UAI'06. Available online at http://ssli.ee.washington.edu/ bilmes/uai06InferenceEvaluation/.

Breiman, L. (1968). Probability. Addison-Wesley. 
Casella, G. and Robert, C. P. (1996). Rao-Blackwellisation of sampling schemes. Biometrika, 83(1):81-94.

Chavira, M. D., Darwiche, A., and Jaeger, M. (2006). Compiling relational Bayesian networks for exact inference. International Journal of Approximate Reasoning, 42(1-2):4-20.

Cheng, J. (2001). Sampling algorithms for estimating the mean of bounded random variables. In Computational Statistics, pages 1-23. Volume 16(1).

Dagum, P. and Luby, M. (1993). Approximating probabilistic inference in Bayesian belief networks is NP-hard. Artificial Intelligence, 60(1):141-153.

Dagum, P. and Luby, M. (1997). An optimal approximation algorithm for Bayesian inference. Artificial Intelligence, 93:1-27.

Darwiche, A., Dechter, R., Choi, A., Gogate, V., and Otten, L. (2008). Results from the Probablistic Inference Evaluation of UAI'08. Available online at: http://graphmod.ics.uci.edu/uai08/Evaluation/Report.

Davies, J. and Bacchus, F. (2008). Distributional importance sampling for approximate weighted model counting. Workshop on Counting Problems in CSP and SAT, and other neighbouring problems.

Dechter, R. (1999). Bucket elimination: A unifying framework for reasoning. Artificial Intelligence, 113:41-85.

Dechter, R., Gogate, V., Otten, L., Marinescu, R., and Mateescu, R. (2009). Graphical model algorithms at UC Irvine. website: http://graphmod.ics.uci.edu/group/Software.

Dechter, R., Kask, K., Bin, E., and Emek, R. (2002a). Generating random solutions for constraint satisfaction problems. In Proceedings of the Eighteenth National Conference on Artificial Intelligence (AAAI), pages 15-21.

Dechter, R., Kask, K., and Mateescu, R. (2002b). Iterative join graph propagation. In Proceedings of the Eighteenth Conference in Uncertainty in Artificial Intelligence, pages $128-136$.

Elidan, G. and Globerson, A. (2010). The 2010 uai approximate inference challenge. Avaliable at http://www.cs.huji.ac.il/project/UAI10/.

Fieguth, P. W., Karl, W. C., and Willsky, A. S. (1998). Efficient multiresolution counterparts to variational methods for surface reconstruction. Computer Vision and Image Understanding, 70(2):157-176.

Fishelson, M. and Geiger, D. (2003). Optimizing exact genetic linkage computations. In Proceedings of the seventh annual international conference on Research in computational molecular biology, pages 114-121.

Geweke, J. (1989). Bayesian inference in econometric models using Monte Carlo integration. Econometrica, 57(6):1317-39.

Gogate, V. (2009). Sampling Algorithms for Probabilistic Graphical Models with Determinism. PhD thesis, Computer Science, University of California, Irvine, USA. 
Gogate, V., Bidyuk, B., and Dechter, R. (2007). Studies in lower bounding probability of evidence using the Markov inequality. In Proceedings of the Twenty Third Conference on Uncertainty in Artificial Intelligence, pages 141-148.

Gogate, V. and Dechter, R. (2005). Approximate inference algorithms for hybrid Bayesian networks with discrete constraints. In Proceedings of the Twenty First Annual Conference on Uncertainty in Artificial Intelligence, pages 209-216.

Gogate, V. and Dechter, R. (2007). Samplesearch: A scheme that searches for consistent samples. Proceedings of the Eleventh Conference on Artificial Intelligence and Statistics, pages $147-154$.

Gogate, V. and Dechter, R. (2011). SampleSearch: Importance sampling in presence of determinism. Artificial Intelligence, 175(2):694-729.

Gomes, C. and Shmoys, D. (2002). Completing quasigroups or latin squares: A structured graph coloring problem. In Proceedings of the Computational Symposium on Graph Coloring and Extensions.

Gomes, C. P., Hoffmann, J., Sabharwal, A., and Selman, B. (2007). From sampling to model counting. In Proceedings of the Twentieth International Joint Conference on Artificial Intelligence, pages 2293-2299.

Hoeffding, W. (1963). Probability inequalities for sums of bounded random variables. Journal of the American Statistical Association, 58(301):13-30.

Kaplan, H. M. (1987). A method of one-sided nonparametric inference for the mean of a nonnegative population. The American Statistician, 41(2):157-158.

Kroc, L., Sabharwal, A., and Selman, B. (2008). Leveraging Belief Propagation, Backtrack Search, and Statistics for Model Counting. In Fifth International Conference on Integration of $A I$ and OR Techniques in Constraint Programming for Combinatorial Optimization Problems, pages 127-141.

Larrosa, J. and Dechter, R. (2003). Boosting search with variable elimination in constraint optimization and constraint satisfaction problems. Constraints, 8(3):303-326.

Leisink, M. and Kappen, B. (2003). Bound propagation. Journal of Artificial Intelligence Research, 19:139-154.

Li, F. and Perona, P. (2005). A Bayesian hierarchical model for learning natural scene categories. In Computer Vision and Pattern Recognition (CVPR), volume 2, pages 524531.

Liao, L., Patterson, D. J., Fox, D., and Kautz, H. (2007). Learning and inferring transportation routines. Artificial Intelligence, 171(5-6):311-331.

Liu, J. (2001). Monte-Carlo strategies in scientific computing. Springer-Verlag, New York.

Marshall, A. W. (1956). The use of multi-stage sampling schemes in Monte Carlo computations. In Symposium on Monte Carlo Methods, pages 123-140.

Mateescu, R., Kask, K., Gogate, V., and Dechter, R. (2010). Join-graph propagation algorithms. Journal of Artificial Intelligence Research, 37:279-328. 
Middleton, B., Michael Shwe M., S., Heckerman, D., Harold Lehmann M., D., and Cooper, G. (1991). Probabilistic diagnosis using a reformulation of the internist-1/qmr knowledge base. Medicine, 30:241-255.

Murphy, K. P., Weiss, Y., and Jordan, M. I. (1999). Loopy belief propagation for approximate inference: An empirical study. In In Proceedings of the Fifteenth Conference on Uncertainty in Artificial Intelligence, pages 467-475.

Ott, J. (1999). Analysis of Human Genetic Linkage. The Johns Hopkins University Press, Baltimore, Maryland.

Pavlovic, V., Rehg, J. M., Cham, T.-J., and Murphy, K. P. (1999). A dynamic Bayesian network approach to figure tracking using learned dynamic models. In The Proceedings of the Seventh IEEE International Conference on Computer Vision, volume 1, pages 94-101.

Pradhan, M., Provan, G., Middleton, B., and Henrion, M. (1994). Knowledge engineering for large belief networks. In Proceedings of the 10th Canadian Conference on Artificial Intelligence, pages 484-490.

Raphael, C. (2002). A hybrid graphical model for rhythmic parsing. Artificial Intelligence, 137(1-2):217-238.

Rish, I. and Dechter, R. (2000). Resolution versus search: Two strategies for sat. Journal of Automated Reasoning, 24:225-275.

Ritter, T. (2003). Latin squares: A literature survey. Available online at: http://www.ciphersbyritter.com/RES/LATSQ.HTM.

Rubinstein, R. Y. (1981). Simulation and the Monte Carlo Method. John Wiley \& Sons Inc.

Sang, T., Beame, P., and Kautz, H. A. (2005). Performing bayesian inference by weighted model counting. In Proceedings, The Twentieth National Conference on Artificial Intelligence, pages 475-482.

Selman, B., Kautz, H., and Cohen, B. (1994). Noise strategies for local search. In Proceedings of the Eleventh National Conference on Artificial Intelligence, pages 337-343.

Simon, L., Berre, D. L., and Hirsch, E. (2005). The SAT 2002 competition. Annals of Mathematics and Artificial Intelligence(AMAI), 43:307-342.

Sorensson, N. and Een, N. (2005). Minisat v1.13-a SAT solver with conflict-clause minimization. In SAT 2005 competition.

Valiant, L. G. (1987). The complexity of enumeration and reliability problems. Siam Journal of Computation, 8(3):105-117.

Walsh, T. (2000). SAT v CSP. In Proceedings of the 6th International Conference on Principles and Practice of Constraint Programming, pages 441-456, London, UK. SpringerVerlag.

Walsh, T. (2001). Permutation problems and channelling constraints. In Proceedings of the 8th International Conference on Logic Programming and Automated Reasoning (LPAR), pages $377-391$. 
Wei, W., Erenrich, J., and Selman, B. (2004). Towards efficient sampling: Exploiting random walk strategies. In Proceedings of the Nineteenth National Conference on Artificial Intelligence, pages $670-676$.

Wei, W. and Selman, B. (2005). A new approach to model counting. In Proceedings of Eighth International Conference on Theory and Applications of Satisfiability Testing (SAT), pages 324-339.

Yedidia, J. S., Freeman, W. T., and Weiss, Y. (2004). Constructing free energy approximations and generalized belief propagation algorithms. IEEE Transactions on Information Theory, 51:2282-2312.

Yuan, C. and Druzdzel, M. J. (2006). Importance sampling algorithms for Bayesian networks: Principles and performance. Mathematical and Computer Modeling, 43(910):1189-1207. 